\title{
Gorenstein algebras and algebras with dominant dimension
}

at least 2 .

\author{
M. Auslander* \\ Department of Mathematics \\ Brandeis University \\ Waltham, Mass. 02254-9110
}

USA

\author{
Ø. Solberg ${ }^{\dagger}$ \\ Institutt for matematikk og statistikk \\ Universitetet i Trondheim, AVH \\ N-7055 Dragvoll \\ NORWAY
}

September 15, 2005

\section{Introduction.}

This paper is devoted to showing how one can use the general theory for relative cotilting modules developed in $[6,7]$ to construct artin algebras (i) with dominant dimension at least 2, (ii) Gorenstein algebras and (iii) Gorenstein algebras with dominant dimension at least 2. All algebras $\Lambda$ considered in this paper are artin algebras and $\bmod \Lambda$ denotes the category of all finitely generated left $\Lambda$-modules. All subfunctors $F$ of $\operatorname{Ext}_{\Lambda}^{1}():,(\bmod \Lambda)^{\mathrm{op}} \times \bmod \Lambda \rightarrow \mathrm{Ab}$ are assumed to be additive and have enough projectives and injectives. Moreover, the subcategory of relative projective modules $\mathcal{P}(F)$, therefore also the subcategory of relative injective modules $\mathcal{I}(F)$, is assumed to be of finite type. For any module $M$ in $\bmod \Lambda$ let add $M$ denote the smallest additive subcategory containing $M$.

In this paper we introduce the following class of artin algebras. An artin algebra $\Lambda$ is said to be $D$ Tr-selfinjective if the subcategory $\mathcal{O}_{\Lambda}=\operatorname{add}\left\{(\operatorname{Tr} D)^{i} \Lambda\right\}_{i=0}^{\infty}$ of $\bmod \Lambda$ is of finite type. Algebras of finite type and selfinjective algebras are clearly $D$ Tr-selfinjective. In addition we show that the Auslander algebra of a selfinjective algebra is $D$ Tr-selfinjective. The reason for introducing this class of algebras is that it is used to describe the Gorenstein algebras with both dominant and injective dimension equal to 2 as endomorphism rings of relative cotilting tilting modules over $D$ Tr-selfinjective algebras. Recall that an artin algebra $\Lambda$ is said to be Gorenstein if the injective dimension of $\Lambda$ on both sides is finite. Denote by $\mathcal{T}$ the class of artin Gorenstein algebras with both dominant and injective dimension equal to 2 . Let $\Gamma$ be in $\mathcal{T}$ and let $\Gamma=T \oplus Q$, where $T$ is the maximal injective summand of $\Gamma$. Denote $\operatorname{End}_{\Gamma}(T)$ by $\Lambda$. We show that $\Gamma \simeq \operatorname{End}_{\Lambda}\left({ }_{\Lambda} T\right)$ and that $\Lambda$ is $D$ Tr-selfinjective. Moreover, the subcategory add ${ }_{\Lambda} T$ is the disjoint union of $\mathcal{O}_{\Lambda}$ and add $M$, where $M \simeq D \operatorname{Tr} M$ and the projective $\Lambda$-modules $\mathcal{P}(\Lambda)$ is properly contained in add ${ }_{\Lambda} T$. This gives us an 1-1 correspondence up to Morita equivalence between $\mathcal{T}$ and the class of pairs $(\Lambda, M)$ where $\Lambda$ is $D$ Tr-selfinjective, $M \simeq D \operatorname{Tr} M$ and the projective $\Lambda$-modules are properly contained in $\mathcal{O}_{\Lambda} \cup$ add $M$. The module ${ }_{\Gamma} T$ is a dualizing summand of the cotilting module $\Gamma$ in $\bmod \Gamma($ see section 1 for the definition). In [7] we proved that all cotilting modules with a dualizing summand are induced from relative cotilting modules. This used to prove this correspondence. The inverse of the above correspondence is given by the following. If $C$ is a $\Lambda$-module such that add $C=\mathcal{O}_{\Lambda}$ for a $D$ Tr-selfinjective algebra $\Lambda$, the correspondence is given by $(\Lambda, M) \mapsto \operatorname{End}_{\Lambda}(C \oplus M)$ for a $\Lambda$-module $M$ with $M \simeq D \operatorname{Tr} M$. Let $T=C \oplus M$. This is shown using that $T$ is a relative cotilting tilting module with respect to the subfunctor $F=F_{\text {add } T}$ of $\operatorname{Ext}_{\Lambda}^{1}($, ), which is all the exact sequences which remain exact after applying the functor $\operatorname{Hom}_{\Lambda}(T$,$) .$

*Partially supported by NSF Grant No. DMS-8904594

†Supported by the Norwegian Research Council during the preparation of this paper. 
In particular, the above result implies the following. Let $\Lambda$ be a selfinjective algebra. Suppose $M$ is a nonzero $\Lambda$-module such that $M \simeq D \operatorname{Tr} M$. Then $\Gamma=\operatorname{End}_{\Lambda}(\Lambda \oplus M)$ is in $\mathcal{T}$. Moreover, $\Gamma$ is of infinite global dimension unless all indecomposable $\Lambda$-modules are a direct summand of $\Lambda \oplus M$. In particular, this is applicable to group rings of finite groups over a field, since they are symmetric algebras. For symmetric algebras the Auslander-Reiten translate $D$ Tr is the second syzygy $\Omega_{\Lambda}^{2}$. Group rings of finite groups over a field are known to have modules with periodic syzygies, so that these rings have modules such that $M \simeq D \operatorname{Tr} M$. Let $\Gamma$ be in $\mathcal{T}$ and let $T$ be the maximal injective summand of $\Gamma$. We show that $\Gamma$ corresponds to a pair $(\Lambda, M)$ with $\Lambda$ selfinjective and $M \simeq D \operatorname{Tr} M$ if and only if the set of simples in the top of $T$ is the same as the set of simples in the socle of $T$. An algebra with this property is said to be of type (I).

Let $\Gamma$ be in $\mathcal{T}$ with maximal injective summand $T$. Denote $\operatorname{End}_{\Gamma}(T)$ by $\Lambda$. Then we know that $\operatorname{add}_{\Lambda} T$ is equal to $\mathcal{O}_{\Lambda} \cup$ add $M$, where $M \simeq D \operatorname{Tr} M$. If $M=0$, the algebra $\Gamma$ is said to be minimal or of type (II). Let ${ }^{\perp} \Gamma$ denote the subcategory $\left\{X \in \bmod \Gamma \mid \operatorname{Ext}_{\Gamma}^{i}(X, \Gamma)=0, i>0\right\}$. In [2] this subcategory is shown to be functorially finite and extension closed and by [4] the subcategory ${ }^{\perp} \Gamma$ has left and right almost split maps. We show that for all indecomposable noninjective projective $\Gamma$-module $P$ there exists an exact sequence $0 \rightarrow P \stackrel{f}{\rightarrow} X \stackrel{g}{\rightarrow} P^{\prime}$ in ${ }^{\perp} \Gamma$, where $P^{\prime}$ is an indecomposable projective $\Gamma$-module and $f$ and $g$ are respectively left and right almost split maps in ${ }^{\perp} \Gamma$. Denote $P^{\prime}$ by $\tau_{\perp \Gamma}^{-1} P$. An algebra $\Gamma$ in $\mathcal{T}$ is said to be of type (III) if there is an indecomposable noninjective

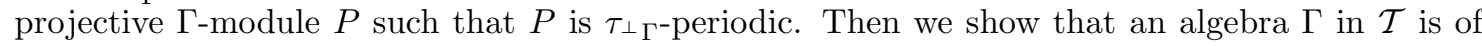
type (I), type (II) or type (III). Moreover, if $\Gamma$ is of type (III) we show that $\Gamma \simeq \operatorname{End}_{\Gamma^{\prime}}\left(\Gamma^{\prime} \oplus M\right)^{\mathrm{op}}$, where $\Gamma^{\prime}$ is of type (II) and $M$ is in ${ }^{\perp} \Gamma^{\prime}$ satisfying $M \simeq \tau_{\perp} \Gamma^{\prime}(M)$. This is the content of the last section of the paper.

In the first section we try to make the paper as selfcontained as possible by giving a very short summary of some of the most frequently used results and definitions from [5, 6, 7]. For a more detailed discussion we refer the reader to those papers. The second section is devoted to constructing algebras of dominant dimension at least 2. In the next section we construct artin Gorenstein algebras as endomorphism rings of relative cotilting modules. In the fourth section we combine the results from the two previous sections and construct artin Gorenstein algebras with dominant dimension at least 2 . The content of the last section is described above.

\section{Preliminaries.}

For the basic definitions and results, as well as, notation concerning relative homology and relative cotilting theory we refer the reader to the papers $[5,6,7]$. However for the convenience of the reader we recall some of the most frequently used definitions and results from those papers.

Let $\Lambda$ be an artin algebra. Let $F$ be an additive subfunctor of $\operatorname{Ext}_{\Lambda}^{1}():,(\bmod \Lambda)^{\mathrm{op}} \times \bmod \Lambda \rightarrow$ Ab. An exact sequence $0 \rightarrow A \rightarrow B \rightarrow C \rightarrow 0$ is called $F$-exact if it is in $F(C, A)$. A module $P$ in $\bmod \Lambda$ is $F$-projective if all $F$-exact sequences ending in $P$ split. An $F$-injective module is defined dually. Let $\mathcal{P}(F)$ and $\mathcal{I}(F)$ denote the subcategory of $F$-projective modules in $\bmod \Lambda$ and the subcategory of $F$-injective modules in $\bmod \Lambda$. These subcategories are connected via the following relations, $\mathcal{P}(F)=\mathcal{P}(\Lambda) \cup \operatorname{Tr} D \mathcal{I}(F)$ and $\mathcal{I}(F)=\mathcal{I}(\Lambda) \cup D \operatorname{Tr} \mathcal{P}(F)$. A subfunctor has enough projectives if for all modules $C$ in $\bmod \Lambda$ there exists an $F$-exact sequence $0 \rightarrow A \rightarrow P \rightarrow C \rightarrow 0$ with $P$ in $\mathcal{P}(F)$. To have enough injectives is defined dually.

Let $\mathcal{X}$ be any subcategory of $\bmod \Lambda$. Define $F_{\mathcal{X}}:(\bmod \Lambda)^{\text {op }} \times \bmod \Lambda \rightarrow$ Sets by for each pair of modules $A$ and $C$ in $\bmod \Lambda$ letting

$$
F_{\mathcal{X}}(C, A)=\{0 \rightarrow A \rightarrow B \rightarrow C \rightarrow 0 \mid(\mathcal{X}, B) \rightarrow(\mathcal{X}, C) \rightarrow 0 \text { is exact }\}
$$

Dually we define $F^{\mathcal{X}}$. It is shown in [5] that $F_{\mathcal{X}}$ and $F^{\mathcal{X}}$ are additive subfunctors of $\operatorname{Ext}_{\Lambda}^{1}():,(\bmod \Lambda)^{\mathrm{op}} \times$ $\bmod \Lambda \rightarrow \mathrm{Ab}$, where two the constructions are related by the following formula, $F_{\mathcal{X}}=F^{D \operatorname{Tr} \mathcal{X}}$. Moreover, $\mathcal{P}\left(F_{\mathcal{X}}\right)=\mathcal{P}(\Lambda) \cup \mathcal{X}$ and $\mathcal{I}\left(F^{\mathcal{X}}\right)=\mathcal{I}(\Lambda) \cup \mathcal{X}$. We will throughout the paper assume that the subfunctors $F$ we consider have enough projectives and injectives and that $\mathcal{P}(F)$ is a finite subcategory of $\bmod \Lambda$. By [5, Corollary 1.13] such a subfunctor $F$ is given by $F=F_{\mathcal{P}(F)}$, where $\mathcal{P}(F)$ is a functorially finite subcategory of $\bmod \Lambda$ containing the projective $\Lambda$-modules. 
Let $F$ be an additive subfunctor of $\operatorname{Ext}_{\Lambda}^{1}(, \quad):(\bmod \Lambda)^{\mathrm{op}} \times \bmod \Lambda \rightarrow \mathrm{Ab}$. Let $\mathcal{C}$ be any subcategory of $\bmod \Lambda$. We denote by ${ }^{\perp} \mathcal{C}$ the subcategory of $\bmod \Lambda$ given by $\{X \in \bmod \Lambda$ | $\operatorname{Ext}_{F}^{i}(X, \mathcal{C})=0$, for all $\left.i>0\right\}$. The subcategory $\mathcal{C}^{\perp}$ is defined dually.

For an $F$-selforthogonal $\Lambda$-module $T$ denote by $\mathcal{X}_{T}$ the subcategory of ${ }^{\perp} T$ whose objects are the $\Lambda$-modules $C$ such that there is an $F$-exact sequence

$$
0 \rightarrow C \rightarrow T_{0} \stackrel{f_{0}}{\rightarrow} T_{1} \rightarrow \cdots \rightarrow T_{n} \stackrel{f_{n}}{\rightarrow} T_{n+1} \rightarrow \cdots
$$

with $T_{i}$ in add $T$ and $\operatorname{Im} f_{i}$ in ${ }^{\perp} T$ for all $i \geq 0$. For a subcategory $\mathcal{C}$ in $\bmod \Lambda$ we denote by $\widehat{\mathcal{C}}$ the subcategory of $\bmod \Lambda$ whose objects are the $\Lambda$-modules $M$ for which there is an $F$-exact sequence

$$
0 \rightarrow C_{n} \rightarrow C_{n-1} \rightarrow \cdots \rightarrow C_{0} \rightarrow M \rightarrow 0
$$

with $C_{i}$ in $\mathcal{C}$. An $F$-selforthogonal module $T$ is called an $F$-cotilting module if id $F<\infty$ and $\mathcal{I}(F)$ is contained in add $T$. An $F$-tilting module is defined dually.

In $\left[6\right.$, Theorem 3.2] we showed that $\mathcal{X}_{T}={ }^{\perp} T$ and that add $T=\mathcal{X}_{T}^{\perp}$ for all $F$-cotilting modules $T$. One of the main results from the relative cotilting theory developed in [6] is the following.

Theorem 1.1 ([6, Theorem 3.13 and Proposition 3.15]) Let $F$ be the additive subfunctor of $\operatorname{Ext}_{\Lambda}^{1}($,$) . Let T$ be an $F$-cotilting module in $\bmod \Lambda$ and let $\Gamma=\operatorname{End}_{\Lambda}(T)$. Then we have the following.

(a) The subcategory $(\mathcal{P}(F), T)=$ add $T_{0}$ for a cotilting module $T_{0}$ over $\Gamma$ with $\operatorname{id}_{\Gamma} T_{0} \leq$ $\max \left\{\operatorname{id}_{F} T, 2\right\}$. The subcategory $\left(\mathcal{X}_{T}, T\right)=\mathcal{X}_{T_{0}}={ }^{\perp} T_{0}$.

(b) The module ${ }_{\Gamma} T$ is a direct summand of a cotilting module $T_{0}$ over $\Gamma$ with add $T_{0}=$ $(\mathcal{P}(F), T), \operatorname{id}_{\Gamma} T_{0} \leq \max \left\{\operatorname{id}_{F} T, 2\right\}$ and $\operatorname{id}_{\Gamma} T \leq \operatorname{id}_{F} T$. Moreover the natural homomorphism $X \rightarrow \operatorname{Hom}_{\Lambda}\left(\operatorname{Hom}_{\Gamma}(X, T), T\right)$ is an isomorphism for all $X$ in $\mathcal{X}_{T_{0}}={ }^{\perp} T_{0}$.

(c) The number of nonisomorphic indecomposable summands of ${ }_{\Lambda} T$ is the same as the number of nonisomorphic modules in $\mathcal{P}(F)$.

Let $M$ be a module over an algebra $\Gamma$. Denote $\operatorname{End}_{\Gamma}(M)$ by $\Lambda$. If the natural homomorphism $A \rightarrow \operatorname{Hom}_{\Lambda}\left(\operatorname{Hom}_{\Gamma}(A, M), M\right)$ is an isomorphism for some $\Gamma$-module $A$, then $M$ is said to dualize the module $A$. The following characterization of dualizing modules is useful. Recall that if $\mathcal{X}$ is a subcategory in $\bmod \Lambda$, then a map $f: C \rightarrow X$ with $X$ in $\mathcal{X}$ is called a left $\mathcal{X}$-approximation if the induced morphism $\operatorname{Hom}_{\Lambda}(X, Y) \rightarrow \operatorname{Hom}_{\Lambda}(C, Y)$ is an epimorphism for all modules $Y$ in $\mathcal{X}$. A right approximation is defined dually.

Proposition 1.2 ([7, Proposition 2.1]) Let $M$ be an arbitrary module in $\bmod \Lambda$ and let $\Gamma=$ $\operatorname{End}_{\Lambda}(M)$. For a module $A$ in $\bmod \Lambda$ the natural homomorphism

$$
\alpha_{A}: A \rightarrow \operatorname{Hom}_{\Gamma}\left(\operatorname{Hom}_{\Lambda}(A, M), M\right)
$$

is an isomorphism if and only there exists an exact sequence

$$
0 \rightarrow A \stackrel{f}{\rightarrow} M^{n} \rightarrow M^{m}
$$

where $f: A \rightarrow M^{n}$ is a left add $M$-approximation.

An important special case of dualizing modules is the following. Assume that $M$ is dualizing a module $A$. If $M$ is a direct summand of $A$, then $M$ is said to be a dualizing summand of $A$.

The last result we recall from the papers $[5,6,7]$ is the following result.

Proposition 1.3 ([7, Proposition 2.7]) Let $T=T^{\prime} \oplus T^{\prime \prime}$ be a cotilting module over an algebra $\Gamma$, where $T^{\prime}$ is a dualizing summand of $T$. Let $\Lambda=\operatorname{End}_{\Gamma}\left(T^{\prime}\right)$ and $F$ the subfunctor of $\operatorname{Ext}_{\Lambda}^{1}($,$) given by F_{\mathrm{Hom}_{\Gamma}\left(\operatorname{add} T, T^{\prime}\right)}$. The module $T^{\prime}$ is an F-cotilting module in $\bmod \Lambda$ with $\operatorname{id}_{F} T^{\prime} \leq \max \left\{\operatorname{id}_{\Gamma} T, 2\right\}$. 


\section{Construction of artin algebras with dominant dimension at least 2 .}

In this section we show that any artin algebra $\Gamma$ with dominant dimension at least 2 is an endomorphism ring of a generator-cogenerator $T$ over some artin algebra $\Lambda$. Moreover, we show that if $\Gamma$ is not selfinjective, then dom.dim $\Gamma \geq r$ if and only if $\operatorname{Ext}_{\Lambda}^{i}(T, T)=0$ for $0<i<r-1$ and $\operatorname{pd}_{\Lambda} T \geq r-1$.

Using the notion of dualizing modules the following two results characterize when an artin algebra $\Gamma$ is the endomorphism ring of some module $T$ over an artin algebra $\Lambda$.

Lemma 2.1 Let $\Gamma$ be an artin algebra and assume that there is a $\Gamma$-module $T$ dualizing $\Gamma$. Denote by $\Lambda$ the artin algebra $\operatorname{End}_{\Gamma}(T)$. Then $\Gamma$ is isomorphic to $\operatorname{End}_{\Lambda}(T)$.

Proof: By the definition of $T$ dualizing $\Gamma$ the natural homomorphism

$$
\Gamma \rightarrow \operatorname{Hom}_{\Lambda}\left(\operatorname{Hom}_{\Gamma}(\Gamma, T), T\right) \simeq \operatorname{End}_{\Lambda}(T)
$$

is an isomorphism. Hence our desired result.

Remark: Let $T$ be a module over an algebra $\Gamma$. Denote $\operatorname{End}_{\Gamma}(T)$ by $\Lambda$. Whenever the natural homomorphism $\Gamma \rightarrow \operatorname{Hom}_{\Lambda}\left(\operatorname{Hom}_{\Gamma}(\Gamma, T), T\right)$ is an isomorphism, we will consider this as an identification and write $\Gamma=\operatorname{End}_{\Lambda}(T)$ in the rest of the paper.

We also have the converse.

Lemma 2.2 Suppose $\Gamma=\operatorname{End}_{\Lambda}(T)$ for some module $T$ over an artin algebra $\Lambda$. Then the module ${ }_{\Gamma} T$ dualizes $\Gamma$.

Proof: Let $\Lambda^{m} \rightarrow \Lambda^{n} \stackrel{f}{\rightarrow} T \rightarrow 0$ be exact. Then the induced sequence $0 \rightarrow \Gamma=(T, T) \stackrel{(f, T)}{\rightarrow}$ $\left(\Lambda^{n}, T\right) \rightarrow\left(\Lambda^{m}, T\right)$ is exact. We want to show that $\Gamma \stackrel{(f, T)}{\rightarrow} T^{n}$ is a left add $T$-approximation. Applying the functor $\operatorname{Hom}_{\Lambda}(,(\Lambda, T))$ to the sequence gives rise to the following commutative diagram

$$
\begin{aligned}
& \begin{array}{cc}
\left(\left(\Lambda^{n}, T\right),(\Lambda, T)\right) & \stackrel{((f, T),(\Lambda, T))}{\longrightarrow} \\
\uparrow \operatorname{Hom}_{\Lambda}(, T) & ((T, T),(\Lambda, T)) \\
& \uparrow \prec \operatorname{Hom}_{\Lambda}(, T)
\end{array} \\
& \left(\Lambda, \Lambda^{n}\right) \quad \stackrel{(\Lambda, f)}{\rightarrow} \quad(\Lambda, T) \quad \rightarrow \quad 0,
\end{aligned}
$$

where $\left(\Lambda, \Lambda^{n}\right) \rightarrow(\Lambda, T) \rightarrow 0$ is exact and where we used that $\operatorname{Hom}_{\Lambda}(C, X)$ is isomorphic to $\operatorname{Hom}_{\Gamma}((X, T),(C, T))$ for all modules $X$ in add $T$ and all modules $C$ in $\bmod \Lambda$. This implies that $\Gamma \rightarrow T^{n}$ is a left add $T$-approximation. By Proposition 1.2 the module $\Gamma^{T}$ dualizes $\Gamma$.

We also need the following general observation, where we refer the reader to section one for the definition of $\mathcal{X}_{T}$.

Lemma 2.3 Let $T$ be an $F$-cotilting module in $\bmod \Lambda$ and let $\Gamma=\operatorname{End}_{\Lambda}(T)$.

(a) Assume that $M$ is a module in $\mathcal{X}_{T}$ such that $\operatorname{Hom}_{\Lambda}(M, T)$ is in $\mathcal{I}(\Gamma)$. Then the module $M$ is in $\mathcal{P}(F)$ and the homomorphism

$$
\operatorname{Hom}_{\Lambda}(, T): \operatorname{Hom}_{\Lambda}(A, C) \rightarrow \operatorname{Hom}_{\Gamma}((C, T),(A, T))
$$

is an isomorphism functorial in both variables for all modules $C$ in $\bmod \Lambda$ and all modules $A$ in $\bmod \Lambda$ such that there is an exact sequence $M^{r} \rightarrow M^{s} \rightarrow A \rightarrow 0$.

(b) Assume that $P$ is a module in $\left(\mathcal{X}_{T}, T\right)$ such that $\operatorname{Hom}_{\Gamma}(P, T)$ is in $\mathcal{I}(\Lambda)$. Then the module $P$ is in $\mathcal{P}(\Gamma)$ and the homomorphism

$$
\operatorname{Hom}_{\Gamma}(, T): \operatorname{Hom}_{\Gamma}(A, C) \rightarrow \operatorname{Hom}_{\Lambda}((C, T),(A, T))
$$


is an isomorphism functorial in both variables for all modules $C$ in $\bmod \Gamma$ and all modules $A$ in $\bmod \Gamma$ such that there is an exact sequence $P^{r} \rightarrow P^{s} \rightarrow A \rightarrow 0$.

(c) If $M$ is a module in $\mathcal{X}_{T}$ having no nonzero projective summands, then the $\Gamma$-module $\operatorname{Hom}_{\Lambda}(M, T)$ does not have any nonzero injective summand.

Proof: (a) Let $M$ be a module in $\mathcal{X}_{T}$ and assume that $\operatorname{Hom}_{\Lambda}(M, T)$ is in $\mathcal{I}(\Gamma)$. By [6, Proposition 3.8] the homomorphism $\operatorname{Hom}_{\Lambda}(, T): \operatorname{Ext}_{F}^{i}(A, C) \rightarrow \operatorname{Ext}_{\Gamma}^{i}((C, T),(A, T))$ is an isomorphism for all modules $A$ and $C$ in $\mathcal{X}_{T}$. Using this isomorphism it follows easily that $\operatorname{Ext}_{F}^{i}(M, C)=0$ for all $i>0$ and all modules $C$ in $\mathcal{X}_{T}$. Since $\widehat{\mathcal{X}_{T}}=\bmod \Lambda$, this implies that $\operatorname{Ext}_{F}^{i}(M)=$,0 and therefore the module $M$ is in $\mathcal{P}(F)$.

So by the above we can assume that $M$ is in $\mathcal{P}(F)$. By [6, Lemma 3.3] the homomorphism

$$
\operatorname{Hom}_{\Lambda}(, T): \operatorname{Hom}_{\Lambda}(A, C) \rightarrow \operatorname{Hom}_{\Gamma}((C, T),(A, T))
$$

is an isomorphism functorial in both variables for all $A$ in $\bmod \Lambda$ and for all $C$ in $\mathcal{X}_{T}$. Let $C$ be an arbitrary module in $\bmod \Lambda$ and let $X_{1} \rightarrow X_{0} \rightarrow C \rightarrow 0$ be $F$-exact with $X_{i}$ in $\mathcal{X}_{T}$ for $i=0,1$. This gives rise to the exact sequence $0 \rightarrow(C, T) \rightarrow\left(X_{0}, T\right) \rightarrow\left(X_{1}, T\right)$. Since $\operatorname{Hom}_{\Lambda}(M, T)$ is injective and $M$ is in $\mathcal{P}(F)$, we obtain the following commutative exact diagram

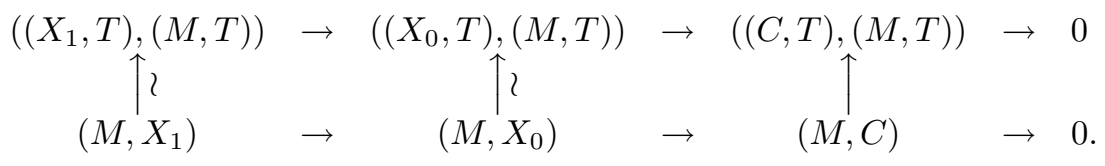

Hence $\operatorname{Hom}_{\Lambda}(, T): \operatorname{Hom}_{\Lambda}(M, C) \rightarrow \operatorname{Hom}_{\Gamma}((C, T),(M, T))$ is an isomorphism. It is easy to see that this isomorphism extends to an isomorphism

$$
\operatorname{Hom}_{\Lambda}(, T): \operatorname{Hom}_{\Lambda}(A, C) \rightarrow \operatorname{Hom}_{\Gamma}((C, T),(A, T))
$$

functorial in both variables for all modules $C$ in $\bmod \Lambda$ and all modules $A$ in $\bmod \Lambda$ such that there exists an exact sequence $M^{r} \rightarrow M^{s} \rightarrow A \rightarrow 0$.

(b) The proof of (b) is similar to the proof of (a) and it is left to the reader.

(c) Let $M$ be a module in $\mathcal{X}_{T}$ having no nonzero projective summands. Assume that $E$ is an injective summand of $\operatorname{Hom}_{\Lambda}(M, T)$ over $\Gamma$. Again using [6, Lemma 3.3] the summand $E$ is isomorphic to $(X, T)$ for some direct summand $X$ of $M$. We want to prove that $X$ is projective. Let $Y \stackrel{f}{\rightarrow} X \rightarrow 0$ be an epimorphism. Since $(X, T)$ is injective, the induced monomorphism $0 \rightarrow(X, T) \rightarrow(Y, T)$ is a split monomorphism. By (a) the splitting is given by $(g, T)$ for some $g: X \rightarrow Y$ and therefore $g$ is also a splitting for $f$. This implies that $X$ is projective. Hence we have a contradiction, so that $\operatorname{Hom}_{\Lambda}(M, T)$ has no nonzero injective summands.

Lemma 2.4 Let $\Lambda$ be an artin algebra and let $T$ be a $\Lambda$-module. Denote by $\Gamma$ the artin algebra $\operatorname{End}_{\Lambda}(T)$ and assume that natural homomorphism $\Lambda \rightarrow \operatorname{End}_{\Gamma}(T)$ is an isomorphism. Then $\operatorname{id}_{\Gamma} T=$ 0 if and only if $\mathcal{I}(\Lambda)$ is contained in $\operatorname{add}_{\Lambda} T$.

Proof: Let $\Lambda$ be an artin algebra and $T$ a $\Lambda$-module. Denote $\operatorname{End}_{\Lambda}(T)$ by $\Gamma$. Assume that $\mathcal{I}(\Lambda)$ is contained in add $T$. Since $\mathcal{I}(\Lambda)$ is contained in add $T$, we have that $T$ is a relative cotilting module with respect to the subfunctor $F=F^{\operatorname{add} T}$ of $\operatorname{Ext}_{\Lambda}^{1}($,$) . Then \operatorname{id}_{F} T=0$ and by Theorem 1.1 (b) we have that $\operatorname{id}_{\Gamma} T=0$.

Conversely, assume that $\operatorname{id}_{\Gamma} T=0$. This is the same as saying that $\operatorname{Hom}_{\Lambda}(\Lambda, T)$ is an injective $\Gamma$-module. We have that

$$
\operatorname{Hom}_{\Lambda}(, T): \operatorname{Hom}_{\Lambda}(A, C) \rightarrow \operatorname{Hom}_{\Gamma}((C, T),(A, T))
$$

is an isomorphism for all modules $A$ in $\bmod \Lambda$ and $C$ in add $T$. Since the module ${ }_{\Lambda} T$ dualizes $\Lambda$ by Lemma 2.2 and by the above isomorphism, it is easy to see that $\operatorname{Hom}_{\Lambda}(\Lambda, P) \simeq$ 
$\operatorname{Hom}_{\Gamma}((P, T),(\Lambda, T))$ for all modules $P$ in $\mathcal{P}(\Lambda)$. Let $A$ be an arbitrary module in $\bmod \Lambda$ and let $P_{1} \rightarrow P_{0} \rightarrow A \rightarrow 0$ be a $\Lambda$-projective presentation of $A$. Then $0 \rightarrow(A, T) \rightarrow\left(P_{0}, T\right) \rightarrow\left(P_{1}, T\right)$ is exact. Since $(\Lambda, T)$ is an injective $\Gamma$-module, the upper row in the following commutative diagram is exact

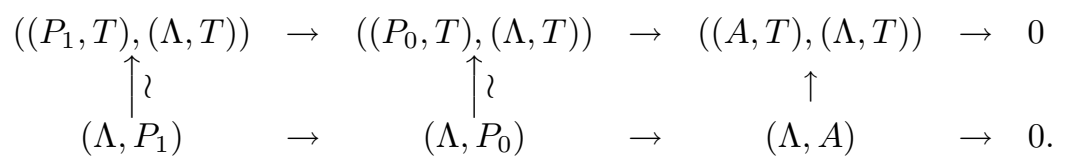

It follows that the natural homomorphism $A \rightarrow \operatorname{Hom}_{\Gamma}\left(\operatorname{Hom}_{\Lambda}(A, T), T\right)$ is an isomorphism for all modules $A$ in $\bmod \Lambda$. By Proposition 1.2 this implies that $\operatorname{Sub}(T)=\bmod \Lambda$ and therefore $\mathcal{I}(\Lambda)$ is contained in $\operatorname{add} T$.

Now we have the necessary preliminaries to show that the endomorphism ring of a generatorcogenerator over any artin algebra has dominant dimension at least 2.

Proposition 2.5 Let $T$ be a generator-cogenerator in $\bmod \Lambda$. Then $\Gamma=\operatorname{End}_{\Lambda}(T)$ has dominant dimension at least 2 and if $I$ is the maximal injective summand of $\Gamma$, then $\operatorname{add} I=\operatorname{add}_{\Gamma} T$.

Proof: Let $T$ be a generator-cogenerator in $\bmod \Lambda$. In particular, this means that $\mathcal{I}(\Lambda)$ is contained in $\operatorname{add} T$. Then $T$ is a relative cotilting module in $\bmod \Lambda$ with respect to the theory given by $F=F^{\text {add } T}$. Let $\Gamma=\operatorname{End}_{\Lambda}(T)$. Since $\operatorname{id}_{F} T=0$, we have that $\operatorname{id}_{\Gamma} T=0$ by Lemma 2.4 or Theorem 1.1.

Because ${ }_{\Lambda} T$ is a generator, $\mathcal{P}(\Lambda)$ is contained in add $T$ and therefore it follows that ${ }_{\Gamma} T$ is in $\mathcal{P}(\Gamma)$. Let $P_{1} \rightarrow P_{0} \rightarrow T \rightarrow 0$ be a $\Lambda$-projective presentation of $T$. Then the induced sequence $0 \rightarrow \Gamma=(T, T) \rightarrow\left(P_{0}, T\right) \rightarrow\left(P_{1}, T\right)$ is exact with $\left(P_{i}, T\right)$ in $\operatorname{add}_{\Gamma} T$. Since ${ }_{\Gamma} T$ is injective and projective, we have that $\operatorname{dom} \cdot \operatorname{dim} \Gamma \geq 2$.

It remains to prove that the maximal injective summand of $\Gamma$ is $\Gamma T$. Since $\operatorname{id}_{F} T=0$, we have that $\mathcal{X}_{T}={ }^{\perp} T=\bmod \Lambda$. The module $\Gamma$ is given by $\operatorname{Hom}_{\Lambda}(T, T)$. It follows from Lemma 2.3 (c) that if $T=P \oplus M$, where $P$ is the maximal projective summand of $T$, then the direct summand $\operatorname{Hom}_{\Lambda}(M, T)$ of $\Gamma$ has no nonzero injective summands. Since ${ }_{\Lambda} T$ is a generator, add $P=\mathcal{P}(\Lambda)$. We have already seen that ${ }_{\Gamma} T$ is injective, so this implies that $\operatorname{add}_{\Gamma} T$ is equal to add $I$ where $I$ is the maximal injective summand of $\Gamma$.

We also have the converse of this proposition.

Proposition 2.6 Let $\Gamma$ be an artin algebra with $\operatorname{dom} \cdot \operatorname{dim} \Gamma \geq 2$. Let $_{\Gamma} T$ be the maximal injective summand of $\Gamma$. Denote $\operatorname{End}_{\Gamma}(T)$ by $\Lambda$. Then ${ }_{\Lambda} T$ is a generator-cogenerator in $\bmod \Lambda$ and $\Gamma=$ $\operatorname{End}_{\Lambda}(T)$.

Proof: Since the module ${ }_{\Gamma} T$ dualizes $\Gamma$, we have that $\Gamma=\operatorname{End}_{\Lambda}(T)$ by Lemma 2.1. Because ${ }_{\Lambda} T=\operatorname{Hom}_{\Gamma}(\Gamma, T)$ and ${ }_{\Gamma} T$ is in $\mathcal{P}(\Gamma)$, it follows that ${ }_{\Lambda} T$ is a generator for $\bmod \Lambda$. By Lemma 2.4 the module ${ }_{\Lambda} T$ is a cogenerator and this completes the proof of the proposition.

Remark: (1) Contrary to what the above results may suggest, the fact that $\Gamma=\operatorname{End}_{\Lambda}(T)$ has dominant dimension at least 2 does not imply that $T$ is a generator-cogenerator for $\bmod \Lambda$, since we have the following. Let $\Lambda$ be an artin algebra and let $I$ be a nonzero twosided ideal in $\Lambda$. Let $T$ be a generator-cogenerator for the artin algebra $\Lambda / I$. Then by Proposition 2.5 the artin algebra $\Gamma=\operatorname{End}_{\Lambda / I}(T)$ has dominant dimension at least 2. We have that $\Gamma=\operatorname{End}_{\Lambda / I}(T)=\operatorname{End}_{\Lambda}(T)$, but $T$ is not a generator-cogenerator for $\bmod \Lambda$.

(2) We can weaken the assumptions in Proposition 2.6 in the following way. Let $T$ be a cogenerator for mod $\Lambda$ such that there exists an exact sequence $P_{1} \rightarrow P_{0} \rightarrow T \rightarrow 0$ with $P_{i}$ in add $T \cap \mathcal{P}(\Lambda)$. Then $\Gamma=\operatorname{End}_{\Lambda}(T)$ has dominant dimension at least 2. We also have the dual statement. We leave the proofs of these results to the reader. 
(3) Now we want to point out how the above constructions are connected to Wedderburn projectives and Wedderburn correspondence. Let $\Gamma$ be an artin algebra with dominant dimension at least 2 . Then there exists an exact sequence $0 \rightarrow \Gamma \rightarrow I_{0} \rightarrow I_{1}$ where $I_{0}$ and $I_{1}$ are projective and injective. Since $I_{j}$ is projective, the module $I_{j}$ is of the form $\operatorname{Hom}_{\Gamma \text { op }}\left(P_{j}, \Gamma^{\mathrm{op}}\right)$ for some projective module $P_{j}$ in $\bmod \Gamma^{\mathrm{op}}$. Because $I_{1}$ is in add $I_{0}$, we also have that $P_{1}$ is in add $P_{0}$. It follows from this that there exists an exact sequence $0 \rightarrow \Gamma \stackrel{f}{\rightarrow} \operatorname{Hom}_{\Gamma_{\mathrm{op}}}\left(P_{0}, \Gamma^{\mathrm{op}}\right)^{n} \rightarrow \operatorname{Hom}_{\Gamma_{\mathrm{op}}}\left(P_{0}, \Gamma^{\mathrm{op}}\right)^{m}$, where $f$ is a left add $\operatorname{Hom}_{\Gamma \text { op }}\left(P_{0}, \Gamma^{\mathrm{op}}\right)$-approximation. By $[7$, Proposition 3.4] this is equivalent to that $P_{0}$ is a Wedderburn projective in $\bmod \Gamma^{\mathrm{op}}$. Let $\Delta=\operatorname{End}_{\Gamma \mathrm{op}}\left(P_{0}\right)^{\mathrm{op}}$ and $G=\operatorname{Hom}_{\Gamma_{\mathrm{op}}}\left(P_{0}, \Gamma^{\mathrm{op}}\right)$. Then $\left(\Gamma^{\mathrm{op}}, P_{0}\right)$ and $(\Delta, G)$ are corresponding Wedderburn pairs. Furthermore, we have that

$$
\Delta=\operatorname{End}_{\Gamma \mathrm{op}}\left(P_{0}\right)^{\mathrm{op}} \simeq \operatorname{End}_{\Gamma}\left(\left(P_{0}, \Gamma^{\mathrm{op}}\right),\left(P_{0}, \Gamma^{\mathrm{op}}\right)\right)=\operatorname{End}_{\Gamma}\left(I_{0}\right)=\Lambda
$$

and

$$
G=\operatorname{Hom}_{\Gamma \mathrm{op}}\left(P_{0}, \Gamma^{\mathrm{op}}\right) \simeq \operatorname{Hom}_{\Gamma}\left(\left(\Gamma^{\mathrm{op}}, \Gamma^{\mathrm{op}}\right),\left(P_{0}, \Gamma^{\mathrm{op}}\right)\right)=\operatorname{Hom}_{\Gamma}\left(\Gamma, I_{0}\right)={ }_{\Lambda} I_{0} .
$$

If $T$ is the maximal injective summand of $\Gamma$, then $\operatorname{add} T=\operatorname{add} I_{0}$. Hence, $\left(\Gamma^{\mathrm{op}}, P_{0}\right)$ and $\left(\Lambda,{ }_{\Lambda} I_{0}\right)$ are corresponding Wedderburn pairs, where the latter is Morita equivalent to $\left(\Lambda^{\prime}=\operatorname{End}_{\Gamma}(T), \Lambda^{\prime} T\right)$.

Let $\Gamma$ be an artin algebra with $\operatorname{dom} \cdot \operatorname{dim} \Gamma=r \geq 2$. Let $T$ be the maximal injective summand of $\Gamma$ and $\Lambda=\operatorname{End}_{\Gamma}(T)$. Now we want to characterize the property that dom.dim $\Gamma=r$ in terms of properties of $T$ as a module over $\Lambda$.

Proposition 2.7 Let $\Gamma$ be an artin algebra with dom.dim $\Gamma \geq 2$ and not selfinjective. Let $T$ be the maximal injective summand of $\Gamma$ and let $\Lambda=\operatorname{End}_{\Gamma}(T)$. Then $\operatorname{dom} \cdot \operatorname{dim} \Gamma \geq r$ if and only if $\operatorname{Ext}_{\Lambda}^{i}(T, T)=0$ for $0<i<r-1$ and $\operatorname{pd}_{\Lambda} T \geq r-1$.

Proof: Let $\Gamma$ be an artin algebra with $\operatorname{dom} \cdot \operatorname{dim} \Gamma=r \geq 2$ and not selfinjective. Let $T$ and $\Lambda$ be as given in the proposition. Then there exists an exact sequence

$$
0 \rightarrow \Gamma \rightarrow\left(P_{0}, T\right) \rightarrow \cdots \rightarrow\left(P_{r-1}, T\right) \rightarrow I_{r} \rightarrow I_{r+1} \rightarrow \cdots
$$

where $P_{i}$ is in $\mathcal{P}(\Lambda)$ for $i=0,1, \ldots, r-1$ and $I_{j}$ is injective for $j \geq r$. This sequence is induced from a sequence of maps in $\bmod \Lambda, \cdots \rightarrow P_{1} \rightarrow P_{0} \rightarrow T \rightarrow 0$. The module ${ }_{\Gamma} T=\operatorname{Hom}_{\Lambda}(\Lambda, T)$ is injective, such that upper row in the following commutative diagram

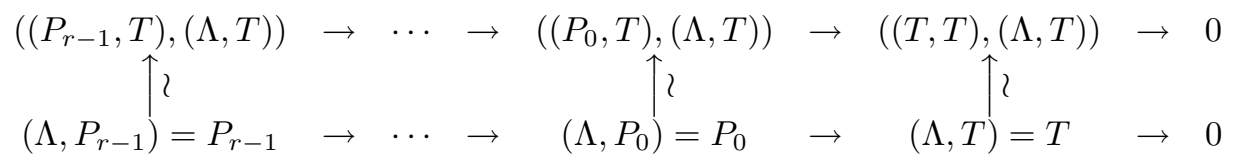

is exact. Therefore the sequence $P_{r-1} \rightarrow \cdots \rightarrow P_{0} \rightarrow T \rightarrow 0$ is exact. Since the sequence $0 \rightarrow(T, T) \rightarrow\left(P_{0}, T\right) \rightarrow\left(P_{1}, T\right) \rightarrow \cdots$ is exact for $i \leq r-1$, it follows that $\operatorname{Ext}_{\Lambda}^{i}(T, T)=0$ for $0<i<r-1$. If $\operatorname{pd}_{\Lambda} T<r-1$, then it follows that $\operatorname{dom} \cdot \operatorname{dim} \Gamma<r$. This is a contradiction, so that $\operatorname{pd}_{\Lambda} T \geq r-1$.

Conversely, assume that $\operatorname{Ext}_{\Lambda}^{i}(T, T)=0$ for $0<i<r-1$ and $\operatorname{pd}_{\Lambda} T \geq r-1$. Let $\cdots \rightarrow$ $P_{r-1} \rightarrow \cdots \rightarrow P_{0} \rightarrow T \rightarrow 0$ be a $\Lambda$-projective resolution of $T$. Applying the functor $\operatorname{Hom}_{\Lambda}(, T)$ to this sequence gives rise to the exact sequence $0 \rightarrow \Gamma \rightarrow\left(P_{0}, T\right) \rightarrow \cdots \rightarrow\left(P_{r-1}, T\right)$. Therefore dom.dim $\Gamma \geq r$.

\section{Construction of Gorenstein algebras.}

This section is devoted to giving a way of constructing artin Gorenstein algebras as endomorphism rings of relative cotilting modules. Recall that an artin algebra $\Lambda$ is said to be Gorenstein if $\operatorname{id}_{\Lambda} \Lambda$ and $\operatorname{id}_{\Lambda^{\mathrm{op}}} \Lambda^{\mathrm{op}}$ both are finite. This is equivalent to the subcategory $\mathcal{P}^{\infty}(\Lambda)$ of $\bmod \Lambda$ consisting of the modules of finite projective dimension being the same as the subcategory $\mathcal{I}^{\infty}(\Lambda) \operatorname{of} \bmod \Lambda$ 
consisting of the modules of finite injective dimension. Throughout this section let $F$ be a subfunctor of $\operatorname{Ext}_{\Lambda}^{1}($,$) . We generalize the notion of Gorenstein algebras to the relative setting. An$ artin algebra $\Lambda$ is said to be $F$-Gorenstein if the subcategories $\mathcal{P}^{\infty}(F)$ and $\mathcal{I}^{\infty}(F)$ are equal. We show that if $T$ is an $F$-cotilting or an $F$-tilting module in $\bmod \Lambda$, then $\Lambda$ is $F$-Gorenstein if and only $\Gamma=\operatorname{End}_{\Lambda}(T)$ is Gorenstein. A main step in the proof of this result, is to show that if $T$ is an $F$-cotilting $F$-tilting module in $\bmod \Lambda$, then $\Gamma=\operatorname{End}_{\Lambda}(T)$ is a Gorenstein algebra.

Finally recall that if $\Lambda$ is Gorenstein, then it is well-known that $\operatorname{id}_{\Lambda} \Lambda=\operatorname{id}_{\Lambda^{\text {op }}} \Lambda^{\text {op }}$ or equivalently that $\operatorname{id}_{\Lambda} \Lambda=\operatorname{pd}_{\Lambda} D\left(\Lambda^{\mathrm{op}}\right)$. So if $\Lambda$ is Gorenstein, we only need to find $\operatorname{id}_{\Lambda} \Lambda$. We will also show that this is true for $F$-Gorenstein algebras.

Proposition 3.1 Let $T$ be an F-cotilting F-tilting module for $\bmod \Lambda$. Then $\Gamma=\operatorname{End}_{\Lambda}(T)$ is an artin Gorenstein algebra with $\operatorname{id}_{\Gamma} \Gamma \leq \operatorname{pd}_{F} T+\max \left\{\operatorname{id}_{F} T, 2\right\}$.

Proof: Assume that $T$ is an $F$-cotilting $F$-tilting module in $\bmod \Lambda$. Denote $\operatorname{End}_{\Lambda}(T)$ by $\Gamma$. Since $T$ is an $F$-tilting module, there exists an $F$-exact sequence

$$
0 \rightarrow P \rightarrow T_{0} \rightarrow T_{1} \rightarrow \cdots \rightarrow T_{n} \rightarrow 0
$$

with $T_{i}$ in add $T$, where add $P=\mathcal{P}(F)$ and $n \leq \operatorname{pd}_{F} T$. Because $T$ is $F$-selforthogonal, the induced sequence

$$
0 \rightarrow\left(T_{n}, T\right) \rightarrow \cdots \rightarrow\left(T_{1}, T\right) \rightarrow\left(T_{0}, T\right) \rightarrow(P, T) \rightarrow 0
$$

is exact and $\operatorname{pd}_{\Gamma}(P, T) \leq n$. The module $T^{\prime}=\operatorname{Hom}_{\Lambda}(P, T)$ is a cotilting module in $\bmod \Gamma$ by Theorem 1.1, therefore $\mathcal{I}(\Gamma)$ is contained in a $\widehat{\operatorname{dd} T^{\prime}}$. Then there exists an exact sequence

$$
0 \rightarrow T_{m}^{\prime} \rightarrow \cdots \rightarrow T_{1}^{\prime} \rightarrow T_{0}^{\prime} \rightarrow D\left(\Gamma^{\mathrm{op}}\right) \rightarrow 0
$$

with $T_{i}^{\prime}$ in add $T^{\prime}$ and $m \leq \operatorname{id}_{\Gamma} T^{\prime}$. From this it follows that $\operatorname{id}_{\Gamma^{\mathrm{op}}} \Gamma^{\mathrm{op}} \leq \operatorname{pd}_{F} T+\max \left\{\operatorname{id}_{F} T, 2\right\}$, since $\operatorname{id}_{\Gamma} T^{\prime} \leq \max \left\{\operatorname{id}_{F} T, 2\right\}$.

Since ${ }_{\Lambda} T$ is an $F$-tilting module, there is an $F$-exact sequence

$$
0 \rightarrow P_{n} \rightarrow \cdots \rightarrow P_{1} \rightarrow P_{0} \rightarrow T \rightarrow 0
$$

with $P_{i}$ in $\mathcal{P}(F)$. Again, using that $T$ is an $F$-selforthogonal module the induced sequence

$$
0 \rightarrow \Gamma=(T, T) \rightarrow\left(P_{0}, T\right) \rightarrow\left(P_{1}, T\right) \rightarrow \cdots \rightarrow\left(P_{n}, T\right) \rightarrow 0
$$

is exact. Because $\left(P_{i}, T\right)$ is in add $T^{\prime}$ for all $i$ and $\operatorname{id}_{\Gamma} T^{\prime}$ is finite, we have that $\operatorname{id}_{\Gamma} \Gamma$ is finite. Therefore $\Gamma$ is an artin Gorenstein algebra.

This result raises two natural questions. When does $\Lambda$ have an $F$-cotilting $F$-tilting module? Which artin Gorenstein algebras do we obtain by this construction? In order to answer the first question we make the following definition and observation. An artin algebra $\Lambda$ is said to be $F$-Gorenstein if $\mathcal{I}^{\infty}(F)=\mathcal{P}^{\infty}(F)$.

Lemma 3.2 (a) An artin algebra $\Lambda$ is F-Gorenstein if and only if both the relative injective dimension of all modules in $\mathcal{P}(F)$ and the relative projective dimension of all modules in $\mathcal{I}(F)$ are bounded.

(b) Let $\Lambda$ be an $F$-Gorenstein algebra. Then $\max \left\{\operatorname{pd}_{F} I \mid I \in \mathcal{I}(F)\right\}$ is equal to $\max \left\{\operatorname{id}_{F} P \mid\right.$ $P \in \mathcal{P}(F)\}$. Furthermore, if $r=\max \left\{\operatorname{id}_{F} P \mid P \in \mathcal{P}(F)\right\}$, then

$$
\mathcal{P}^{\infty}(F)=\mathcal{P}^{r}(F)=\mathcal{I}^{r}(F)=\mathcal{I}^{\infty}(F) .
$$


Proof: (a) Assume that $\Lambda$ is $F$-Gorenstein. Then $\mathcal{P}^{\infty}(F)=\mathcal{I}^{\infty}(F)$. Since $\mathcal{P}(F)$ is of finite type and $\mathcal{P}(F)$ is contained in $\mathcal{I}^{\infty}(F)$, we have that $\max \left\{\operatorname{id}_{F} P \mid P \in \mathcal{P}(F)\right\}$ is finite. Similarly we show that $\max \left\{\operatorname{pd}_{F} I \mid I \in \mathcal{I}(F)\right\}$ is finite.

Conversely, assume that both $r=\max \left\{\operatorname{id}_{F} P \mid P \in \mathcal{P}(F)\right\}$ and $s=\max \left\{\operatorname{pd}_{F} I \mid I \in \mathcal{I}(F)\right\}$ are finite. We have that if $0 \rightarrow A \rightarrow B \rightarrow C \rightarrow 0$ is an $F$-exact sequence, then $\operatorname{pd}_{F} A \leq \operatorname{pd}_{F}(B \oplus C)$ and $\operatorname{id}_{F} C \leq \operatorname{id}_{F}(A \oplus B)$. Let $M$ be a module in $\mathcal{P}^{\infty}(F)$. We want to show that it in in $\mathcal{I}^{\infty}(F)$. Since $M$ is in $\mathcal{P}^{\infty}(F)$, there exists an $F$-exact sequence $0 \rightarrow P_{n} \rightarrow \cdots \rightarrow P_{1} \rightarrow P_{0} \rightarrow M \rightarrow 0$ with $P_{i}$ in $\mathcal{P}(F)$ for some finite $n$. Using the above observation about injective dimensions, it follows that $\operatorname{id}_{F} M \leq r$ and in particular $M$ is in $\mathcal{I}^{\infty}(F)$. By the dual arguments we have that if $N$ is in $\mathcal{I}^{\infty}(F)$, then $\operatorname{pd}_{F} N \leq s$ and in particular in $\mathcal{P}^{\infty}(F)$. This show that $\mathcal{P}^{\infty}(F)=\mathcal{I}^{\infty}(F)$ and $\Lambda$ is $F$-Gorenstein.

(b) Let $\Lambda$ be $F$-Gorenstein and let $r$ and $s$ be as above. Then we have that $\operatorname{Ext}_{F}^{i}(, P)=0$ for all $i \geq r+1$ and all modules $P$ in $\mathcal{P}(F)$ and $\operatorname{Ext}_{F}^{i}(I)=$,0 for all $i \geq s+1$ and all modules $I$ in $\mathcal{I}(F)$. By assumption we have that $\operatorname{Ext}_{F}^{s}(I, P) \neq 0$ for some module $I$ in $\mathcal{I}(F)$ with id $F=s$ and some module $P$ in $\mathcal{P}(F)$. This implies that $s \leq r$. Dually, we get that $r \leq s$ and therefore $r=s$.

Now we characterize when an algebra has an $F$-cotilting $F$-tilting module.

Proposition 3.3 (a) An artin algebra $\Lambda$ has an F-cotilting F-tilting module if and only if $\Lambda$ is F-Gorenstein.

(b) An artin algebra $\Lambda$ is $F$-Gorenstein if and only if a $\Lambda$-module $T$ is an $F$-cotilting module if and only if $T$ is an F-tilting module.

Proof: (a) Assume that $T$ is an $F$-cotilting $F$-tilting module in $\bmod \Lambda$. By the definitions of $F$-cotilting and $F$-tilting modules every module in $\mathcal{I}(F)$ has a finite $F$-exact resolution in add $T$ and every module in $\mathcal{P}(F)$ has a finite $F$-exact coresolution in add $T$. Since $\operatorname{id}_{F} T$ and $\operatorname{pd}_{F} T$ both are finite, it follows immediately that both $\operatorname{pd}_{F} \mathcal{I}(F)$ and $\operatorname{id}_{F} \mathcal{P}(F)$ are finite. Therefore $\Lambda$ is $F$-Gorenstein.

Assume that $\Lambda$ is $F$-Gorenstein. Then both $\mathcal{I}(F)$ and $\mathcal{P}(F)$ are the additive subcategory generated by some $F$-cotilting $F$-tilting module (not necessarily the same module).

(b) Assume that $\Lambda$ is $F$-Gorenstein and that $T$ is an $F$-cotilting module in $\bmod \Lambda$. Since $T$ is $F$-selforthogonal, $\operatorname{id}_{F} T$ is finite and $\Lambda$ is $F$-Gorenstein, it suffices to show that every module in $\mathcal{P}(F)$ has a finite $F$-exact coresolution in add $T$ to prove that $T$ is an $F$-tilting module.

Since $T$ is an $F$-cotilting module, we have that $\mathcal{P}(F)$ is contained in $\mathcal{X}_{T}={ }^{\perp} T$. Therefore there exists an $F$-exact sequence

$$
0 \rightarrow P \rightarrow T_{0} \stackrel{f_{0}}{\rightarrow} T_{1} \stackrel{f_{1}}{\rightarrow} \cdots \rightarrow T_{n} \stackrel{f_{n}}{\rightarrow} T_{n+1} \rightarrow \cdots
$$

for all module $P$ in $\mathcal{P}(F)$, where $T_{i}$ is in $\operatorname{add} T$ and $X_{i}=\operatorname{Im} f_{i}$ is in $\mathcal{X}_{T}$ for all $i \geq 0$. Let $\operatorname{id}_{F} \mathcal{P}(F) \leq r$. Then

$$
\operatorname{Ext}_{F}^{1}\left(X_{r}, X_{r-1}\right)=\operatorname{Ext}_{F}^{2}\left(X_{r}, X_{r-2}\right)=\cdots=\operatorname{Ext}_{F}^{r+1}\left(X_{r}, P\right)=0,
$$

hence the $F$-exact sequence $0 \rightarrow X_{r-1} \rightarrow T_{r} \rightarrow X_{r} \rightarrow 0$ splits, such that $X_{r-1}$ is in add $T$. This implies that $T$ is an $F$-tilting module. Using the dual arguments we can show that if $T$ is an $F$-tilting module, then $T$ is an $F$-cotilting module.

Assume that a $\Lambda$-module $T$ is an $F$-cotilting module if and only if $T$ is an $F$-tilting module. Since a $\Lambda$-module $T$ such that add $T=\mathcal{P}(F)$ is an $F$-tilting module, $T$ is also an $F$-cotilting module. By (a) the algebra $\Lambda$ is $F$-Gorenstein. This completes the proof of the proposition.

As in the classical theory we say that an $F$-cotilting module $T$ is a $r$ - $F$-cotilting module if $\operatorname{id}_{F} T \leq r$. Let $\Lambda$ be an $F$-Gorenstein algebra. Choose a $\Lambda$-module $T$ such that add $T=\mathcal{I}(F)$. Then $T$ is an $F$-cotilting module. Since $\Lambda$ is $F$-Gorenstein, the module $T$ is also an $F$-tilting module. In the classic notation the module $T$ is a $1-F$-cotilting module. We have that $\operatorname{pd}_{F} T=$ $\max \left\{\operatorname{pd}_{F} I \mid I \in \mathcal{I}(F)\right\}$, so that a $1-F$-cotilting module need not be a $1-F$-tilting module. However, we have the following results. 
Proposition 3.4 Let $\Lambda$ be an F-Gorenstein algebra. Suppose $T$ is an F-cotilting module. Then $\operatorname{pd}_{F} \mathcal{I}(F)=\operatorname{id}_{F} \mathcal{P}(F) \leq \operatorname{pd}_{F} T+\operatorname{id}_{F} T$.

Proof: Since $\Lambda$ is $F$-Gorenstein and $T$ is an $F$-cotilting module, the module $T$ is also an $F$-tilting module by Proposition 3.6 (b). Therefore there exists an $F$-exact sequence

$$
0 \rightarrow P \rightarrow T_{0} \stackrel{f_{0}}{\rightarrow} T_{1} \stackrel{f_{1}}{\rightarrow} \cdots \stackrel{f_{n-1}}{\rightarrow} T_{n} \rightarrow 0
$$

for all modules $P$ in $\mathcal{P}(F)$ with $T_{i}$ in add $T$. Denote $\operatorname{Ker} f_{i}$ by $X_{i}$. Assume that $n$ is minimal. For any module $X$ in ${ }^{\perp} T$ we have that

$$
\operatorname{Ext}_{F}^{j}\left(X, X_{i}\right) \simeq \operatorname{Ext}_{F}^{j+1}\left(X, X_{i-1}\right)
$$

for $j \geq 1$. In particular,

$$
\operatorname{Ext}_{F}^{1}\left(T_{n}, X_{n-1}\right) \simeq \operatorname{Ext}_{F}^{2}\left(T_{n}, X_{n-2}\right) \simeq \cdots \simeq \operatorname{Ext}_{F}^{n}\left(T_{n}, P\right)
$$

So, if $n>\operatorname{pd}_{F} T$, then the chosen sequence is not minimal. Therefore we have that $n \leq \operatorname{pd}_{F} T$. If $0 \rightarrow A \rightarrow B \rightarrow C \rightarrow 0$ is $F$-exact, then $\operatorname{id}_{F} A \leq \max \left\{\operatorname{id}_{F} B, \operatorname{id}_{F} C\right\}+1$. From this it follows that $\operatorname{id}_{F} P \leq n+\operatorname{id}_{F} T \leq \operatorname{pd}_{F} T+\operatorname{id}_{F} T$. This is true for all modules $P$ in $\mathcal{P}(F)$, hence $\operatorname{id}_{F} \mathcal{P}(F) \leq \operatorname{id}_{F} T+\operatorname{pd}_{F} T$. Since $\operatorname{id}_{F} \mathcal{P}(F)=\operatorname{pd}_{F} \mathcal{I}(F)$ for $F$-Gorenstein rings, the proof of the proposition is complete.

The following corollary follows immediately from this result.

Corollary 3.5 (a) Let $\Lambda$ be $F$-Gorenstein with $\operatorname{id}_{F} \mathcal{P}(F)=\operatorname{pd}_{F} \mathcal{I}(F)=n$. If $T$ is an r-F-tilting module, then $T$ is an $s$-F-cotilting module where $n-r \leq s \leq n$.

(b) Let $\Lambda$ be Gorenstein with $\operatorname{id}_{\Lambda} \Lambda=n$. If $T$ is an $r$-tilting module, then $T$ is an s-cotilting module where $n-r \leq s \leq n$.

In Proposition 3.1 we saw that if $T$ is an $F$-cotilting $F$-tilting module, then $\Gamma=\operatorname{End}_{\Lambda}(T)$ is Gorenstein. We also have the converse.

Proposition 3.6 Let $\Gamma$ be an artin algebra and assume that $\Gamma=\operatorname{End}_{\Lambda}(T)$ for some $F$-cotilting module $T$ over an artin algebra $\Lambda$. Then the following are equivalent

(a) The artin algebra $\Gamma$ is Gorenstein.

(b) The $\Lambda$-module $T$ is an F-tilting module.

(c) The artin algebra $\Lambda$ is F-Gorenstein.

Proof: The implication (b) implies (a) is Proposition 3.1. By Proposition 3.3 it follows directly that (b) and (c) are equivalent. It remains to prove that (a) implies (b).

Assume that $\Gamma$ is Gorenstein. We want to prove that ${ }_{\Lambda} T$ is an $F$-tilting module in $\bmod \Lambda$. By [6, Proposition 3.7] the extension groups $\operatorname{Ext}_{\Gamma}^{i}(C, A)$ and $\operatorname{Ext}_{\Gamma}^{i}((A, T),(C, T))$ are isomorphic for all $i>0$ and all modules $A$ and $C$ in $\mathcal{X}_{T}={ }^{\perp} T$. In particular we have that

$$
\operatorname{Ext}_{F}^{i}(T, A) \simeq \operatorname{Ext}_{\Gamma}^{i}((A, T), \Gamma)=0
$$

for all $i>\operatorname{id}_{\Gamma} \Gamma$ and all modules $A$ in $\mathcal{X}_{T}$. Since $\mathcal{X}_{T}$-resdim$F(\bmod \Lambda)$ is finite, this implies that $\operatorname{Ext}_{F}^{i}(T)=$,0 for all $i>\operatorname{id}_{\Gamma} \Gamma$ and therefore $\operatorname{pd}_{F} T \leq \operatorname{id}_{\Gamma} \Gamma$.

For an artin Gorenstein algebra $\Gamma$ we have that $\mathcal{I}^{\infty}(\Gamma)=\mathcal{P}^{\infty}(\Gamma)$. Let $P$ a $\Lambda$-module such that add $P=\mathcal{P}(F)$. Then $\operatorname{Hom}_{\Lambda}(P, T)=T^{\prime}$ is a cotilting module over $\Gamma$, and we have the following inclusions,

$$
\mathcal{I}(\Gamma) \subset \widehat{\operatorname{add} T} T^{\prime} \subset \mathcal{I}^{\infty}(\Gamma)=\mathcal{P}^{\infty}(\Gamma)
$$

Therefore $\operatorname{pd}_{\Gamma} T^{\prime}$ is finite and there exists an exact sequence

$$
0 \rightarrow\left(T_{n}, T\right) \rightarrow \cdots \rightarrow\left(T_{1}, T\right) \rightarrow\left(T_{0}, T\right) \rightarrow(P, T)=T^{\prime} \rightarrow 0
$$


in $\bmod \Gamma$ with $T_{i}$ in $\operatorname{add}{ }_{\Lambda} T$. Because all modules in this sequence are in $\left(\mathcal{X}_{T}, T\right)={ }^{\perp} T^{\prime}$, this sequence is induced from an $F$-exact sequence

$$
0 \rightarrow P \rightarrow T_{0} \rightarrow T_{1} \rightarrow \cdots \rightarrow T_{n} \rightarrow 0
$$

in $\bmod \Lambda$. The module $P$ is chosen such that add $P=\mathcal{P}(F)$, hence ${ }_{\Lambda} T$ is an $F$-tilting module, since ${ }_{\Lambda} T$ is $F$-selforthogonal and $\operatorname{pd}_{F} T$ is finite.

Using the above result and its dual, we have the following corollary.

Corollary 3.7 Let $T$ be an F-tilting or F-cotilting module. Then $\Lambda$ is F-Gorenstein if and only if $\Gamma=\operatorname{End}_{\Lambda}(T)$ is Gorenstein.

Now we return to the second question posed above, namely, which artin Gorenstein algebras are obtained as an endomorphism ring of a relative cotilting tilting module which is not a standard cotilting tilting module? Let $\Gamma$ be a Gorenstein algebra. The module $\Gamma^{\mathrm{op}}$ is a cotilting tilting module in mod $\Gamma^{\mathrm{op}}$. We have that $\Gamma=\operatorname{End}_{\Gamma^{\mathrm{op}}}\left(\Gamma^{\mathrm{op}}\right)$. Therefore the restriction that the relative cotilting tilting module should be not a standard cotilting tilting module in the above question, is necessary to make it nontrivial. Let $T$ be an $F$-cotilting $F$-tilting module in $\bmod \Lambda$ and let $\Gamma=\operatorname{End}_{\Lambda}(T)$. Assume that $\Gamma$ is selfinjective. Since the module ${ }_{\Gamma} T$ dualizes $\Gamma$ and $\Gamma$ is injective, $\Gamma$ must be in $\operatorname{add}_{\Gamma} T$. Since ${ }_{\Gamma} T$ is a direct summand of a cotilting module and $\Gamma$ is in $\operatorname{add}_{\Gamma} T$, it follows that add ${ }_{\Gamma} T=\mathcal{P}(\Gamma)$. Therefore the number of nonisomorphic indecomposable modules in $\mathcal{P}(F)$ is the same as the number of nonisomorphic indecomposable projective $\Lambda$-modules. This implies that $\mathcal{P}(F)=\mathcal{P}(\Lambda)$. This shows that if $\Gamma$ is selfinjective and an endomorphism ring of a relative cotilting module $T$ over some algebra $\Lambda$, then the module ${ }_{\Lambda} T$ is a standard cotilting module and $\Lambda$ is Morita equivalent to $\Gamma^{\mathrm{op}}$.

By Proposition 3.3 the cotilting module $T^{\prime}$ in the proof of (a) implies (b) in Proposition 3.6 above is also a tilting module in $\bmod \Gamma$. The above construction gives all artin Gorenstein algebras where there exists a cotilting (and tilting) module $T^{\prime}$ with a proper dualizing summand $T$ of $T^{\prime}$. We do not know the answer to the above question in general, but we have the following partial answer.

Proposition 3.8 Let $\Gamma$ be an artin Gorenstein algebra and assume that in a minimal injective copresentation $0 \rightarrow \Gamma \rightarrow I_{0} \rightarrow I_{1}$ the subcategory $\operatorname{add}\left(I_{0} \oplus I_{1}\right)$ is not all of $\mathcal{I}(\Gamma)$. Then $\Gamma$ is the endomorphism ring of a relative nonstandard cotilting tilting module. Namely, let $T$ be a module such that $\operatorname{add} T=\operatorname{add}\left(I_{0} \oplus I_{1}\right)$ and $\Lambda=\operatorname{End}_{\Gamma}(T)$. Then $\Gamma \simeq \operatorname{End}_{\Lambda}(T)$ and ${ }_{\Lambda} T$ is a relative nonstandard cotilting tilting module with respect to the subfunctor $F=F^{\text {add }} \Lambda^{T}$ of $\operatorname{Ext}_{\Lambda}^{1}($,$) .$

Proof: Let $\Gamma$ be an artin Gorenstein algebra and assume that in a minimal injective copresentation $0 \rightarrow \Gamma \rightarrow I_{0} \rightarrow I_{1}$ the subcategory add $\left(I_{0} \oplus I_{1}\right)$ is not all of $\mathcal{I}(\Gamma)$. Let $T$ be a $\Gamma$-module such that $\operatorname{add} T=\operatorname{add}\left(I_{0} \oplus I_{1}\right)$ and let $\Lambda=\operatorname{End}_{\Lambda}(T)$. By Proposition 1.2 the module $T$ dualizes $\Gamma$ and therefore $\Gamma \simeq \operatorname{End}_{\Lambda}(T)$. Since $\operatorname{id}_{\Gamma} T=0$, the module ${ }_{\Lambda} T$ is a cogenerator for $\bmod \Lambda$ by Lemma 2.4. Let $F=F^{\text {add }{ }_{\Lambda} T}$, then ${ }_{\Lambda} T$ is an $F$-cotilting module. Because $\Gamma$ is Gorenstein and ${ }_{\Lambda} T$ is an $F$-cotilting module, the algebra $\Lambda$ is $F$-Gorenstein by Corollary 3.7. Hence by Proposition 3.3 the module ${ }_{\Lambda} T$ is also an $F$-tilting module and this completes the proof of the proposition.

\section{Construction of Gorenstein algebras with dominant di- mension at least 2 .}

This section is devoted to constructing artin Gorenstein algebras with dominant dimension at least 2 using relative cotilting theory. Let $T$ be a generator-cogenerator in $\bmod \Lambda$ and assume that $T$ is a relative cotilting module with respect to the subfunctor $F=F_{\text {add } T}$ of $\operatorname{Ext}_{\Lambda}^{1}($,$) . Then$ 
$\Gamma=\operatorname{End}_{\Lambda}(T)$ is an artin Gorenstein algebra $\Gamma$ with dominant dimension at least 2. Conversely, we show that any artin Gorenstein algebra $\Gamma$ with $\operatorname{dom} \cdot \operatorname{dim} \Gamma \geq 2$ is obtained in this way. least 2 .

We start by giving the construction of artin Gorenstein algebras with dominant dimension at

Proposition 4.1 Let $\Lambda$ be an artin algebra and $T$ a generator-cogenerator for $\bmod \Lambda$. Assume that $T$ is a relative cotilting module with respect to the relative theory given by the subfunctor $F=F_{\text {add } T}$ of $\operatorname{Ext}_{\Lambda}^{1}($,$) . Then \Gamma \simeq \operatorname{End}_{\Lambda}(T)$ is an artin Gorenstein algebra with dom.dim $\Gamma \geq 2$ and $\operatorname{id}_{\Gamma} \Gamma \leq \max \left\{\operatorname{id}_{F} T, 2\right\}$. Moreover, if $I$ is the maximal injective summand of $\Gamma$, then $\operatorname{add}_{\Gamma} T=$ add $I$.

Proof: This is a combination of Proposition 2.5 and Proposition 3.1.

Next we show that all artin Gorenstein algebras $\Gamma$ with $\operatorname{dom} \cdot \operatorname{dim} \Gamma \geq 2$ are given by the construction in the above result.

Proposition 4.2 Let $\Gamma$ be an artin Gorenstein algebra with $\operatorname{dom} \cdot \operatorname{dim} \Gamma \geq 2$. Let $T$ be the maximal injective summand of $\Gamma$ and let $\Lambda=\operatorname{End}_{\Gamma}(T)$. Then $\Gamma \simeq \operatorname{End}_{\Lambda}(T)$ and $T$ is a generatorcogenerator for mod $\Lambda$ and a relative cotilting module over $\Lambda$ with respect to the subfunctor $F=$ $F_{\text {add }_{\Lambda} T}$ of $\operatorname{Ext}_{\Lambda}^{1}($,$) . Moreover, \operatorname{id}_{F} T \leq \max \left\{\operatorname{id}_{\Gamma} \Gamma, 2\right\}$.

Proof: Let $\Gamma$ be an artin Gorenstein algebra with $\operatorname{dom} \cdot \operatorname{dim} \Gamma \geq 2$ and ${ }_{\Gamma} T$ the maximal injective summand of $\Gamma$. Denote $\operatorname{End}_{\Gamma}(T)$ by $\Lambda$. By Proposition 2.6 the module ${ }_{\Lambda} T$ is a generatorcogenerator for $\bmod \Lambda$ and $\Gamma \simeq \operatorname{End}_{\Lambda}(T)$.

Since $\Gamma$ is Gorenstein, the module $\Gamma$ is a cotilting module in $\bmod \Gamma$ by Proposition 3.6 (b). Because dom.dim $\Gamma \geq 2$, there is an exact sequence $0 \rightarrow \Gamma \rightarrow I_{0} \rightarrow I_{1}$ where $I_{0}$ and $I_{1}$ are in add $T$. Because $T$ is injective, the map $\Gamma \rightarrow I_{0}$ is a left add $T$-approximation. Then Proposition 1.2

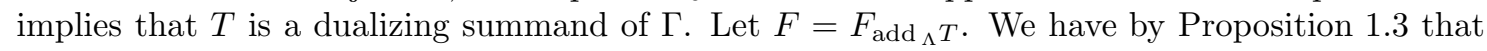
$T$ is an $F$-cotilting module with $\operatorname{id}_{F} T \leq \max \left\{\operatorname{id}_{\Gamma} \Gamma, 2\right\}$.

Remark: It follows from the two previous results that all artin Gorenstein algebras $\Gamma$ with dom.dim $\Gamma=\operatorname{id}_{\Gamma} \Gamma=2$ are obtained as an endomorphism ring of a relative cotilting tilting module $T$ with respect to the subfunctor $F=F_{\text {add } T}$ of $\operatorname{Ext}_{\Lambda}^{1}($,$) and \operatorname{id}_{F} T \leq 2$. We discuss this special case in more detail in the next section.

Next we want to discuss to what extent the relative cotilting module $T$ over the artin algebra $\Lambda$ is unique.

Let $\Gamma$ be an artin Gorenstein algebra with $\operatorname{dom} \cdot \operatorname{dim} \Gamma \geq 2$. Let $\Lambda^{\prime}$ and $T^{\prime}$ be such that $T^{\prime}$ is a relative cotilting module over $\Lambda^{\prime}$ for which $\Gamma \simeq \operatorname{End}_{\Lambda^{\prime}}(T)$. Then the module $T^{\prime}$ is a module over $\Gamma$. So $\Gamma$ is the algebra over which we can compare all relative cotilting modules $T^{\prime}$ having the property $\Gamma \simeq \operatorname{End}_{\Lambda^{\prime}}\left(T^{\prime}\right)$.

Proposition 4.3 Let $\Gamma$ be an artin Gorenstein algebra with $\operatorname{dom} \cdot \operatorname{dim} \Gamma \geq 2$. Then there is a unique artin algebra $\Lambda$ and a unique relative cotilting module $T$ over $\Lambda$, such that $\Gamma \simeq \operatorname{End}_{\Lambda}(T)$, where the module $\Gamma_{\Gamma} T$ has the following property. If $\Lambda^{\prime}$ is an artin algebra and $T^{\prime}$ is a relative cotilting module in $\bmod \Lambda^{\prime}$ with $\Gamma \simeq \operatorname{End}_{\Lambda^{\prime}}\left(T^{\prime}\right)$, then the length of $T^{\prime}$ as a module over $\Gamma$ is less than or equal to the length of $T$ as a module over $\Gamma$.

Proof: Let $\Gamma$ be an artin Gorenstein algebra with $\operatorname{dom} \cdot \operatorname{dim} \Gamma \geq 2$. Assume that $\Lambda^{\prime}$ is an artin algebra and $T^{\prime}$ is a relative cotilting module over $\Lambda^{\prime}$ such that $\Gamma \simeq \operatorname{End}_{\Lambda^{\prime}}\left(T^{\prime}\right)$. It follows from Theorem 1.1 that $T^{\prime}$ as a module over $\Gamma$ dualizes all the modules in $\operatorname{Hom}_{\Lambda^{\prime}}\left(\mathcal{X}_{T^{\prime}}, T^{\prime}\right)$. In particular, ${ }_{\Gamma} T^{\prime}$ dualizes $\Gamma$. By Proposition 1.2 there is an exact sequence $0 \rightarrow \Gamma \rightarrow\left(T^{\prime}\right)^{n} \rightarrow\left(T^{\prime}\right)^{m}$. Let $T$ be the maximal injective summand of $\Gamma$. Then from the above exact sequence we have that add $T$ must be contained in $\operatorname{add}_{\Gamma} T^{\prime}$. We have already seen that if $\Lambda=\operatorname{End}_{\Gamma}(T)$, then $T$ is a relative 
cotilting module over $\Lambda$ such that $\Gamma \simeq \operatorname{End}_{\Lambda}(T)$. Let $I$ be a basic module such that add $I=\operatorname{add} T$. Let $\Lambda=\operatorname{End}_{\Gamma}(I)$. Then ${ }_{\Lambda} I$ is a relative cotilting module over $\Lambda$ such that $\Gamma \simeq \operatorname{End}_{\Lambda}(I)$. Since every relative cotilting module $T^{\prime}$ with $\Gamma \simeq \operatorname{End}_{\Lambda^{\prime}}\left(T^{\prime}\right)$ has add $T$ contained in $\operatorname{add}_{\Gamma} T^{\prime}$, it follows that $\Lambda$ is the unique artin algebra and $I$ is the unique relative cotilting module over $\Lambda$ such that $\Gamma \simeq \operatorname{End}_{\Lambda}(I)$ and the length of $I$ as a module over $\Gamma$ is minimal.

Artin algebras $\Gamma$ with finite global dimension and $\operatorname{dom} \cdot \operatorname{dim} \Gamma \geq 2$ are in particular artin Gorenstein algebras with dominant dimension at least 2. Suppose that $\Gamma$ has finite global dimension with dom.dim $\Gamma \geq 2$. Then we know that $\Gamma=\operatorname{End}_{\Lambda}(T)$ for a relative cotilting tilting $T$ over an artin algebra $\Lambda$. Now we describe what new restrictions $\Gamma$ being of finite global dimension imposes on $\Lambda$ and $T$.

Proposition 4.4 Let $\Gamma$ be an artin algebra of finite global dimension and dom.dim $\Gamma \geq 2$. Let $T$ be the maximal injective summand of $\Gamma$ and let $\Lambda=\operatorname{End}_{\Gamma}(T)$. Then ${ }_{\Lambda} T$ is a generator-cogenerator for $\bmod \Lambda$ and a relative cotilting module over $\Lambda$ with respect to the subfunctor $F=F_{\operatorname{add}_{\Lambda} T}$ of $\operatorname{Ext}_{\Lambda}^{1}($,$) . Moreover, the module { }_{\Lambda} T$ has the following properties

(i) $\operatorname{id}_{F} T \leq \max \left\{\operatorname{id}_{\Gamma} \Gamma, 2\right\}$.

(ii) For every module $C$ in $\bmod \Lambda$ there exists an exact sequence

$$
0 \rightarrow T_{n} \rightarrow T_{n-1} \rightarrow \cdots \rightarrow T_{1} \rightarrow T_{0} \rightarrow C \rightarrow 0
$$

with $T_{i}$ in $\operatorname{add} T$ and $n \leq \operatorname{gl} \cdot \operatorname{dim} \Gamma-2$, where the sequence is a succession of right $\operatorname{add} T$ approximations.

(iii) For every module $A$ in $\bmod \Lambda$ there exists an exact sequence

$$
0 \rightarrow A \rightarrow T_{0} \rightarrow T_{1} \rightarrow \cdots \rightarrow T_{m-1} \rightarrow T_{m} \rightarrow 0
$$

with $T_{i}$ in $\operatorname{add} T$ and $m \leq \operatorname{gl} \cdot \operatorname{dim} \Gamma-2$, where the sequence is a succession of left $\operatorname{add} T$-approximations.

Proof: We only have to prove the statements (ii) and (iii), since the rest was shown in Proposition 4.2 .

(ii) By [6, Proposition 4.1] we have that $\operatorname{gl} \cdot \operatorname{dim}_{F} \Lambda \leq \operatorname{gl} \cdot \operatorname{dim} \Gamma+\operatorname{id}_{F} T$. Since $T$ is $F$-projective and the number of nonisomorphic indecomposable summands of ${ }_{\Lambda} T$ is the same as the number of nonisomorphic indecomposable modules in $\mathcal{P}(F)$ by Theorem 1.1 (c), it follows that add $T=\mathcal{P}(F)$. Hence for each module $C$ in $\bmod \Lambda$ there exists an exact sequence

$$
0 \rightarrow T_{n} \rightarrow T_{n-1} \rightarrow \cdots \rightarrow T_{1} \rightarrow T_{0} \rightarrow C \rightarrow 0
$$

with $T_{i}$ in add $T$ which is a succession of right add $T$-approximations. Applying the functor $\operatorname{Hom}_{\Lambda}(T$,$) gives rise to a \Gamma^{\mathrm{op}}$-projective resolution of $(T, C)$ in $\bmod \Gamma^{\mathrm{op}}$. Since $T$ is a cogenerator, there exists an exact sequence $0 \rightarrow C \rightarrow T_{0}^{\prime} \rightarrow T_{1}^{\prime}$ with $T_{i}^{\prime}$ in add $T$ for $i=0,1$. This induces an exact sequence $0 \rightarrow(T, C) \rightarrow\left(T, T_{0}^{\prime}\right) \rightarrow\left(T, T_{1}^{\prime}\right)$. Therefore every $\Gamma^{\mathrm{op}}$-module of the form $(T, C)$ is a second syzygy. Hence, $\operatorname{pd}_{\Gamma^{\mathrm{op}}}(T, C) \leq$ gl.dim $\Gamma^{\mathrm{op}}-2$. Since gl.dim $\Gamma^{\mathrm{op}}=$ gl.dim $\Gamma$ is finite, we have that $n \leq$ gl.dim $\Gamma-2$.

(iii) Let $A$ be an arbitrary module in $\bmod \Lambda$. Since add $T$ contains $\mathcal{I}(\Lambda)$, every left add $T$ approximation of $A$ is a monomorphism. Therefore there exists an exact sequence

$$
0 \rightarrow A \rightarrow T_{0} \rightarrow T_{1} \rightarrow T_{2} \rightarrow T_{3} \rightarrow \cdots
$$

with $T_{i}$ in add $T$, which is a succession of left add $T$-approximations. By applying the functor $\operatorname{Hom}_{\Lambda}(, T)$ to this sequence we obtain a $\Gamma$-projective resolution of $(A, T)$ in $\bmod \Gamma$. Since $T$ is a generator, there exists an exact sequence $T_{1}^{\prime} \rightarrow T_{0}^{\prime} \rightarrow A \rightarrow 0$ with $T_{i}^{\prime}$ in add $T$ for $i=0,1$. This induces an exact sequence $0 \rightarrow(A, T) \rightarrow\left(T_{0}^{\prime}, T\right) \rightarrow\left(T_{1}^{\prime}, T\right)$ and therefore every $\Gamma$-module of the form $(A, T)$ is a second syzygy. Hence $\operatorname{pd}_{\Gamma}(A, T) \leq$ gl.dim $\Gamma-2$. Since gl.dim $\Gamma$ is finite the above sequence can be chosen to be finite $0 \rightarrow A \rightarrow T_{0} \rightarrow T_{1} \rightarrow \cdots \rightarrow T_{m-1} \rightarrow T_{m} \rightarrow 0$ with $m \leq \operatorname{gl} \cdot \operatorname{dim} \Gamma-2$. 
Remark: If gl.dim $\Gamma=2$, then $\operatorname{gl} \cdot \operatorname{dim}_{F} \Lambda=0$ and therefore add ${ }_{\Lambda} T=\mathcal{P}(F)=\bmod \Lambda$. Hence $\Lambda$ is of finite type and $\Gamma$ is Morita equivalent to the Auslander algebra of $\Lambda$. This gives another way of viewing the well-known result that $\Gamma$ is an Auslander algebra if and only if $\operatorname{dom} \cdot \operatorname{dim} \Gamma=$ gl. $\operatorname{dim} \Gamma=2$.

\section{$5 \quad F$-selfinjective algebras.}

It follows from the previous section that all artin Gorenstein algebras $\Gamma$ with $\operatorname{dom} \cdot \operatorname{dim} \Gamma=\mathrm{id}_{\Gamma} \Gamma=$ 2 are obtained as an endomorphism ring of a relative cotilting tilting module $T$ over some algebra $L$ with respect to the subfunctor $F=F_{\text {add } T}$ of $\operatorname{Ext}_{\Lambda}^{1}($,$) , where \operatorname{id}_{F} T \leq 2$. In this section we show an even stronger result. Namely that they all can be obtained as an endomorphism ring of a relative cotilting tilting module $T$ with respect to the subfunctor $F=F_{\text {add } T}$ of $\operatorname{Ext}_{\Lambda}^{1}($,$) ,$ where $\mathcal{P}(F)=\mathcal{I}(F)$. This naturally leads us to the following definition. Let $F$ be a subfunctor of $\operatorname{Ext}_{\Lambda}^{1}($,$) . An artin algebra \Lambda$ is said to be $F$-selfinjective if $\mathcal{P}(F)=\mathcal{I}(F)$ and $\mathcal{P}(F)$ is a subcategory of finite type. Clearly all selfinjective algebras and all algebras of finite type are $F$-selfinjective for the some $F$ (choose $F=\operatorname{Ext}_{\Lambda}^{1}($,$) and F=0$, respectively). In addition, we show that the Auslander algebra of a selfinjective algebra is $F$-selfinjective for some $F$. It is shown that $\Lambda$ is $F$-selfinjective if and only if the subcategory $\mathcal{O}_{\Lambda}=\operatorname{add}\left\{(\operatorname{Tr} D)^{i} \Lambda\right\}_{i=0}^{\infty}$ is of finite type. Moreover, if $\Lambda$ is $F$-selfinjective, then $\mathcal{P}(F)=\mathcal{I}(F)=\mathcal{O}_{\Lambda} \cup$ add $M$, where $M \simeq D \operatorname{Tr} M$.

Again, let $\Gamma$ be a Gorenstein algebra with $\operatorname{dom} \cdot \operatorname{dim} \Gamma=\operatorname{id}_{\Gamma} \Gamma=2$. Then $\Gamma=\operatorname{End}_{\Lambda}(T)$ for some relative cotilting tilting module $T$ over an $F$-selfinjective algebra $\Lambda$. From the above we have that $\mathcal{P}(F)=\mathcal{I}(F)=\mathcal{O}_{\Lambda} \cup$ add $M$, where $M \simeq D \operatorname{Tr} M$ and $\mathcal{P}(\Lambda)$ is properly contained in $\mathcal{P}(F)$. Then we have three possibilities: (I) $\mathcal{O}_{\Lambda}=\mathcal{P}(\Lambda)$, (II) the module $M$ is zero and (III) the module $M$ is nonzero and $\mathcal{P}(\Lambda)$ is properly contained in $\mathcal{O}_{\Lambda}$. In case (I) the algebra $\Lambda$ is selfinjective and we show that the algebras $\Gamma$ having this property is characterized by the set of simples in the top and the socle of the maximal injective summand of $\Gamma$ coincide. The cases (II) and (III) are also characterized in terms of properties of modules in $\bmod \Gamma$. Thus giving a structure theorem for the artin Gorenstein algebras $\Gamma$ with dom.dim $\Gamma=\operatorname{id}_{\Gamma} \Gamma=2$.

First we characterize when $\Lambda$ is $F$-selfinjective.

Proposition 5.1 (a) An artin algebra $\Lambda$ is $F$-selfinjective for some subfunctor $F$ of $\operatorname{Ext}_{\Lambda}^{1}($, ) if and only if one of the following statements is true for $\Lambda$.

(i) The subcategory add $\left\{(D \operatorname{Tr})^{i} D\left(\Lambda^{\mathrm{op}}\right),(\operatorname{Tr} D)^{i} \Lambda\right\}_{i=0}^{\infty}$ of $\bmod \Lambda$ is of finite type.

(ii) $\operatorname{add}\left\{(D \operatorname{Tr})^{i} D\left(\Lambda^{\mathrm{op}}\right)\right\}_{i=0}^{\infty}=\operatorname{add}\left\{(\operatorname{Tr} D)^{i} \Lambda\right\}_{i=0}^{\infty}$.

(iii) The subcategory add $\left\{(D \operatorname{Tr})^{i} D\left(\Lambda^{\mathrm{op}}\right)\right\}_{i=0}^{\infty}$ is of finite type.

(iv) The subcategory add $\left\{(\operatorname{Tr} D)^{i} \Lambda\right\}_{i=0}^{\infty}$ is of finite type.

(b) If $\Lambda$ is F-selfinjective, then

$$
\mathcal{P}(F)=\mathcal{I}(F)=\mathcal{O}_{\Lambda} \cup \text { add } M
$$

where $\mathcal{O}_{\Lambda}=\operatorname{add}\left\{(\operatorname{Tr} D)^{i} \Lambda\right\}_{i=0}^{\infty}$ is of finite type and $M \simeq D \operatorname{Tr} M$.

Proof: (a) First we show that $\Lambda$ is $F$-selfinjective for some subfunctor $F$ if and only if statement (i) is true.

Assume that $\Lambda$ is $F$-selfinjective for some subfunctor $F$ of $\operatorname{Ext}_{\Lambda}^{1}($,$) . We have that \mathcal{I}(F)=$ $\mathcal{I}(\Lambda) \cup D \operatorname{Tr} \mathcal{P}(F)$ and $\mathcal{P}(F)=\mathcal{P}(\Lambda) \cup \operatorname{Tr} D(\mathcal{I}(F))$. Since $\mathcal{P}(F)=\mathcal{I}(F)$, it follows that $(D \operatorname{Tr})^{i} D\left(\Lambda^{\mathrm{op}}\right)$ and $(\operatorname{Tr} D)^{i} \Lambda$ are in $\mathcal{P}(F)=\mathcal{I}(F)$ for all $i \geq 0$. Therefore the subcategory $\mathcal{O}_{\Lambda}=\operatorname{add}\left\{(D \operatorname{Tr})^{i} D\left(\Lambda^{\mathrm{op}}\right),(\operatorname{Tr} D)^{i} \Lambda\right\}_{i=0}^{\infty}$ of $\bmod \Lambda$ is contained in $\mathcal{P}(F)$. Since $\mathcal{P}(F)$ is of finite type, the subcategory $\mathcal{O}_{\Lambda}$ is also of finite type.

Conversely, assume that the subcategory

$$
\mathcal{O}_{\Lambda}=\operatorname{add}\left\{(D \operatorname{Tr})^{i} D\left(\Lambda^{\mathrm{op}}\right),(\operatorname{Tr} D)^{i} \Lambda\right\}_{i=0}^{\infty}
$$


is of finite type. Let $F=F_{\mathcal{O}_{\Lambda}}$. Since $\mathcal{P}(F)=\mathcal{P}(\Lambda) \cup \mathcal{O}_{\Lambda}$ and $\mathcal{I}(F)=\mathcal{I}(\Lambda) \cup D \operatorname{Tr} \mathcal{P}(F)$, it follows that $\mathcal{P}(F)=\mathcal{I}(F)$ by the definition of the subcategory $\mathcal{O}_{\Lambda}$. Therefore we have that $\Lambda$ is $F$-selfinjective.

It is not hard to see that all the statements in (i)-(iv) are equivalent.

(b) Assume that $\Lambda$ is $F$-selfinjective. By the proof of (a) the subcategory $\mathcal{O}_{\Lambda}=\operatorname{add}\left\{(\operatorname{Tr} D)^{i} \Lambda\right\}_{i=0}^{\infty}$ is contained in $\mathcal{P}(F)$. Therefore the subcategory $\mathcal{O}_{\Lambda}$ is of finite type. Assume that $X$ is a nonzero indecomposable module in $\mathcal{P}(F) \backslash \mathcal{O}_{\Lambda}$. As above it follows that $(D \operatorname{Tr})^{i} X$ is in $\mathcal{P}(F)=\mathcal{I}(F)$ for all $i \geq 0$. Since $(D \operatorname{Tr})^{i} X \neq 0$ for all $i \geq 0$ and $(D \operatorname{Tr})^{i} X$ is not in $\mathcal{O}_{\Lambda}$ for any $i \geq 0$, then $(D \operatorname{Tr})^{i} X$ must be isomorphic to $X$ for some $i_{0}$. Therefore $X$ is a direct summand of the module $M=\oplus_{i=0}^{i_{0}-1}(D \operatorname{Tr})^{i} X$ in $\mathcal{P}(F) \backslash \mathcal{O}_{\Lambda}$. The claim in (b) follows immediately from this.

This characterization of $F$-selfinjective algebras naturally suggests the following definition. An artin algebra $\Lambda$ is called $D$ Tr-selfinjective if the subcategory add $\left\{(\operatorname{Tr} D)^{i} \Lambda\right\}_{i=0}^{\infty}$ is of finite type. Then Proposition 5.1 says that an artin algebra $\Lambda$ is $D$ Tr-selfinjective if and only if $\Lambda$ is $F$-selfinjective for some subfunctor $F$ of $\operatorname{Ext}_{\Lambda}^{1}($,$) .$

The two first parts of the following result are immediate consequences of the above result.

Proposition 5.2 (a) Every artin algebra $\Lambda$ of finite type is DTr-selfinjective.

(b) Every artin selfinjective algebra $\Lambda$ is D Tr-selfinjective.

(c) The Auslander algebra of an artin selfinjective algebra is D Tr-selfinjective.

Proof: We only prove (c). Let $\Lambda$ be selfinjective of finite type and let $T$ be a $\Lambda$-module such that

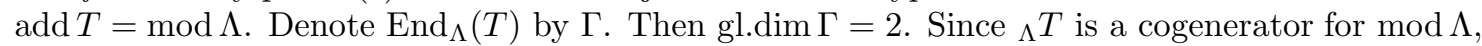
the module ${ }_{\Gamma} T$ is injective by Lemma 2.4. Let $P_{1} \rightarrow P_{0} \rightarrow T \rightarrow 0$ be a $\Lambda$-projective presentation of $T$. Then the induced sequence

$$
0 \rightarrow \Gamma \rightarrow\left(P_{0}, T\right) \rightarrow\left(P_{1}, T\right) \rightarrow I \rightarrow 0
$$

is exact, where the module $\left(P_{i}, T\right)$ is injective and projective for $i=0,1$ and $I$ is injective. Moreover, any indecomposable injective $\Gamma$-module is a direct summand of one of the modules $\left(P_{0}, T\right),\left(P_{1}, T\right)$ and $I$. We want to show that $\operatorname{add}\left\{(D \operatorname{Tr})^{i} \mathcal{I}(\Gamma)\right\}_{i=0}^{\infty}$ is of finite type. Since $\Gamma_{\Gamma}$ is injective and projective, $D \operatorname{Tr} T=0$. The above sequence is a $\Gamma$-projective resolution of $I$. Applying $\operatorname{Hom}_{\Gamma}(, \Gamma)$ to the above sequence gives rise to the following commutative diagram

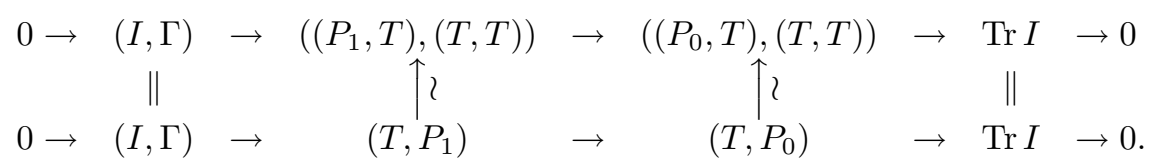

Therefore the sequence $0 \rightarrow D \operatorname{Tr} I \rightarrow D\left(T, P_{0}\right) \rightarrow D\left(T, P_{1}\right) \rightarrow D(I, \Gamma) \rightarrow 0$ is exact. Assume that the module $D\left(T, P_{i}\right)$ is projective for $i=0,1$. Since gl.dim $\Gamma=2$, the module $D \operatorname{Tr} I$ is projective and we are done. So we only need to prove that the module $(T, \Lambda)$ is an injective $\Gamma^{\mathrm{op}}$-module.

Let $C$ be an arbitrary module in $\bmod \Gamma^{\mathrm{op}}$ and let $0 \rightarrow\left(T, T_{2}\right) \rightarrow\left(T, T_{1}\right) \rightarrow\left(T, T_{0}\right) \rightarrow C \rightarrow 0$ be a $\Gamma^{\mathrm{op}}$-projective resolution of $C$. This sequence is induced from a complex $0 \rightarrow T_{2} \rightarrow T_{2} \rightarrow T_{0}$ in $\bmod \Lambda$. Since $T$ is a generator, this complex is exact. Then we have the following commutative diagram

$$
\begin{aligned}
& 0 \rightarrow(C,(T, \Lambda)) \rightarrow\left(\left(T, T_{0}\right),(T, \Lambda)\right) \rightarrow\left(\left(T, T_{1}\right),(T, \Lambda)\right) \quad \rightarrow \quad\left(\left(T, T_{2}\right),(T, \Lambda)\right) \\
& \begin{array}{ccccc}
\uparrow 2 & \uparrow 2 & & \uparrow 2 \\
\left(T_{0}, \Lambda\right) & \rightarrow & \left(T_{1}, \Lambda\right) & \rightarrow & \left(T_{2}, \Lambda\right) \rightarrow 0,
\end{array}
\end{aligned}
$$

where the lower row is exact since $\Lambda$ is injective. Therefore the module $(T, \Lambda)$ is an injective $\Gamma^{\mathrm{op}}$-module and this completes the proof of the proposition.

The next result is a special case of Proposition 4.1. 
Proposition 5.3 Suppose that $\Lambda$ is F-selfinjective. Let $T$ be a module such that add $T$ is equal to $\mathcal{P}(F)=\mathcal{I}(F)$ and assume that $\mathcal{P}(\Lambda)$ is a proper subcategory of $\mathcal{P}(F)$. Then $\Gamma=\operatorname{End}_{\Lambda}(T)$ is a Gorenstein algebra satisfying the following properties.

(i) $\operatorname{dom} \cdot \operatorname{dim} \Gamma=\operatorname{id}_{\Gamma} \Gamma=2$.

(ii) If $I$ is the maximal injective summand of $\Gamma$, then $\operatorname{add}_{\Gamma} T=\operatorname{add} I$.

(iii) The categories $\bmod \Lambda$ and ${ }^{\perp} \Gamma$ are dual via the functors

$$
\operatorname{Hom}_{\Lambda}(, T): \bmod \Lambda \rightarrow \bmod \Gamma
$$

and

$$
\operatorname{Hom}_{\Gamma}(, T): \bmod \Gamma \rightarrow \bmod \Lambda
$$

Proof: The only statement that does not follow from Proposition 4.1 is (iii). In general the functors $\operatorname{Hom}_{\Lambda}(, T): \bmod \Lambda \rightarrow \bmod \Gamma$ and $\operatorname{Hom}_{\Gamma}(, T): \bmod \Gamma \rightarrow \bmod \Lambda$ induce dualities between ${ }_{\Lambda} T$ and ${ }^{\perp} \operatorname{Hom}_{\Lambda}(\mathcal{P}(F), T)$ by $[6$, Corollary $3.6(\mathrm{a})]$. Since $\operatorname{id}_{F} T=0$ and $\operatorname{add}_{\Lambda} T=\mathcal{P}(F)$, we have that ${ }^{\perp} T=\bmod \Lambda$ and that ${ }^{\perp} \operatorname{Hom}_{\Lambda}(\mathcal{P}(F), T)={ }^{\perp} \Gamma$. The claim in (iii) follows from this.

Also the converse of this result is true, but to prove it we need the following lemma.

Lemma 5.4 Let $\Gamma$ be an artin Gorenstein algebra with $\operatorname{dom} \cdot \operatorname{dim} \Gamma=\operatorname{id}_{\Gamma} \Gamma=2$ and let $T$ be the maximal injective summand of $\Gamma$. Then a $\Gamma$-module $X$ is in ${ }^{\perp} \Gamma$ if and only if there exists an exact sequence $0 \rightarrow X \rightarrow T_{0} \rightarrow T_{1}$ with $T_{i}$ in add $T$ for $i=0,1$.

Proof: Let $\Gamma$ be an artin Gorenstein algebra with dom.dim $\Gamma=\operatorname{id}_{\Gamma} \Gamma=2$ and let $T$ be the maximal injective summand of $\Gamma$. Then $T$ dualizes $\Gamma$. We claim that $T$ dualizes all modules in ${ }^{\perp} \Gamma$. Let $\Lambda=\operatorname{End}_{\Gamma}(T)$. Since $\Gamma$ is a cotilting module, there exists an exact sequence $0 \rightarrow X \rightarrow \Gamma^{n} \rightarrow \Gamma^{m}$ for all module $X$ in ${ }^{\perp} \Gamma$. Because ${ }_{\Gamma} T$ is injective, the sequence $\left(\Gamma^{m}, T\right) \rightarrow\left(\Gamma^{n}, T\right) \rightarrow(X, T) \rightarrow 0$ is exact in $\bmod \Lambda$. Then we obtain the following commutative diagram

$$
\begin{array}{cccccc}
0 & \rightarrow \quad((X, T), T) & \rightarrow & \left(\left(\Gamma^{n}, T\right), T\right) & \rightarrow & \left(\left(\Gamma^{m}, T\right), T\right) \\
\uparrow & & \uparrow \imath & & \uparrow \imath \\
0 & \rightarrow \quad X & \rightarrow & \Gamma^{n} & \rightarrow & \Gamma^{m} .
\end{array}
$$

Hence, it follows that $T$ dualizes all modules $X$ in ${ }^{\perp} \Gamma$ and therefore there exists an exact sequence $0 \rightarrow X \rightarrow T_{0} \rightarrow T_{1}$ with $T_{i}$ in add $T$ for $i=0,1$ by Proposition 1.2.

Assume that there exists an exact sequence $0 \rightarrow X \rightarrow T_{0} \rightarrow T_{1}$ for a $\Gamma$-module $X$ with $T_{i}$ in add $T$ for $i=0,1$. Since ${ }_{\Gamma} T$ is projective, it follows that $X$ is a second syzygy of some module in $\bmod \Gamma$. Because $\operatorname{id}_{\Gamma} \Gamma=2$, every second syzygy is in ${ }^{\perp} \Gamma$, therefore $X$ is in ${ }^{\perp} \Gamma$. This completes the proof of the lemma.

Now we can show the following converse of Proposition 5.3.

Proposition 5.5 Let $\Gamma$ be an artin Gorenstein algebra with dom.dim $\Gamma=\mathrm{id}_{\Gamma} \Gamma=2$. Denote the maximal injective summand of $\Gamma$ by $T$ and let $\Lambda=\operatorname{End}_{\Gamma}(T)$. Then $\Gamma=\operatorname{End}_{\Lambda}(T)$ and if $F=F_{\operatorname{add}_{\Lambda} T}$, then $\Lambda$ is $F$-selfinjective and $\mathcal{P}(F)=\mathcal{I}(F)=\operatorname{add}_{\Lambda} T$.

Proof: From Proposition 4.2 we have that $\Gamma=\operatorname{End}_{\Lambda}(T)$ and if $F=F_{\text {add }_{\Lambda} T}$, then $T$ is an $F$ cotilting module with $\operatorname{id}_{F} T \leq 2$. Since the number of nonisomorphic indecomposable summands of ${ }_{\Lambda} T$ is the same as the number of nonisomorphic indecomposable modules in $\mathcal{P}(F)$ (or $\mathcal{I}(F)$ ), it is enough to show that $\operatorname{id}_{F} T=0$ in order to show that $\Lambda$ is $F$-selfinjective.

We have already seen that the functors $\operatorname{Hom}_{\Lambda}(, T): \bmod \Lambda \rightarrow \bmod \Gamma$ and $\operatorname{Hom}_{\Gamma}(, T): \bmod \Gamma \rightarrow$ $\bmod \Lambda$ induce dualities between ${ }^{\perp}{ }_{\Lambda} T$ and ${ }^{\perp} \Gamma$. Let $M$ be an arbitrary module in $\bmod \Lambda$. We want to show that $\operatorname{Hom}_{\Lambda}(M, T)$ is in ${ }^{\perp} \Gamma$. Let $\left(T_{1}, T\right) \rightarrow\left(T_{0}, T\right) \rightarrow M \rightarrow 0$ be a $\Lambda$-projective presentation of $M$. Then $0 \rightarrow(M, T) \rightarrow T_{0} \rightarrow T_{1}$ is exact with $T_{i}$ in $\operatorname{add}_{\Gamma} T$ for $i=0,1$. By Lemma 5.4 the $\Gamma$-module $(M, T)$ is in ${ }^{\perp} \Gamma$. Since clearly all modules of the form $\operatorname{Hom}_{\Gamma}(X, T)$ for 
$X$ in ${ }^{\perp} \Gamma$ are in $\bmod \Lambda$, it follows that $\bmod \Lambda$ and ${ }^{\perp} \Gamma$ are dual via the above functors. Hence ${ }^{\perp} T=\bmod \Lambda$ and therefore $\operatorname{id}_{F} T=0$.

Next we show that the only algebras of finite global dimension we obtain in this way are algebras Morita equivalent to an Auslander algebra of some artin algebra.

Proposition 5.6 Let $\Lambda$ be an F-selfinjective artin algebra and let $T$ be a $\Lambda$-module such that add $T=\mathcal{P}(F)=\mathcal{I}(F)$. Denote $\operatorname{End}_{\Lambda}(T)$ by $\Gamma$. Then the following are equivalent.

(a) gl.dim $\Gamma$ is finite.

(b) $\operatorname{gl} \cdot \operatorname{dim} \Gamma=2$.

(c) $\operatorname{add}_{\Lambda} T=\bmod \Lambda$.

In particular, if gl.dim $\Gamma$ is finite, then $\Lambda$ is of finite type and $\Gamma$ is Morita equivalent to the Auslander algebra of $\Lambda$.

Proof: From [6, Proposition 4.1] we have that gl.dim $\operatorname{dim}_{F} \leq$ gl.dim $\Gamma \leq 2+$ gl.dim ${ }_{F} \Lambda$. We also have that ${ }_{\Lambda} T=\bmod \Lambda$ and therefore

$$
\left({ }_{\Lambda}^{\perp} T\right)^{\perp}=\widehat{\operatorname{add} T}=\operatorname{add} T=\mathcal{P}^{\infty}(F) .
$$

Assume that gl.dim $\Gamma$ is finite. Then gl. $\operatorname{dim}_{F} \Lambda$ is also finite and therefore $\bmod \Lambda=\mathcal{P}^{\infty}(F)=$ add $T$. Hence $\Lambda$ is of finite type and $\Gamma$ is Morita equivalent to the Auslander algebra of $\Lambda$. This show that (a) implies (b) and (c). We clearly have that (b) implies (a) and that (c) implies (a).

Now we characterize the artin algebras $\Gamma$ with Gorenstein dimension and dominant dimension both equal to 2 , which are obtained by the above construction from selfinjective algebras.

Proposition 5.7 Let $\Lambda$ be a selfinjective artin algebra and $M$ a nonzero module in mod $\Lambda$ with $M \simeq D \operatorname{Tr} M$. Let $T=\Lambda \oplus M$. The artin algebra $\Gamma=\operatorname{End}_{\Lambda}(T)$ has the following properties.

(a) The algebra $\Gamma$ is a Gorenstein algebra, where the maximal injective summand of $\Gamma$ is a dualizing summand. Moreover, $\operatorname{id}_{\Gamma} \Gamma=2$.

(b) dom.dim $\Gamma=2$ and dom.dim $\Gamma^{\mathrm{op}}=2$.

(c) If $I$ is the maximal injective summand of $\Gamma$, then $\operatorname{add} \operatorname{soc} I=\operatorname{add} I / \underline{\mathrm{r}} I$.

(d) The functor $\operatorname{Hom}_{\Gamma}(, T):{ }^{\perp} \Gamma \rightarrow \bmod \Lambda$ is a duality.

Proof: The statements (a), half of (b) and (d) follows from Proposition 5.3.

(b) It is known in general that if $\operatorname{dom} \cdot \operatorname{dim} \Gamma=2$, then $\operatorname{dom} \cdot \operatorname{dim} \Gamma^{\mathrm{op}}=2$. Here we give a new proof of this fact. We have that $\Gamma^{\mathrm{op}} \simeq \operatorname{End}_{\Lambda \mathrm{op}}(D(\Lambda) \oplus D(M))$, where $D(\Lambda)$ is selfinjective and $D(M) \simeq D \operatorname{Tr}(D M)$. Applying what we have shown for $\Gamma$ to $\Gamma^{\mathrm{op}}$, it follows that $\operatorname{dom} \cdot \operatorname{dim} \Gamma^{\mathrm{op}}=2$.

(c) The module $\Gamma_{\Gamma} T$ is the maximal injective summand of $\Gamma$. We want to prove that add $\operatorname{soc} T=$ add $T / \underline{\mathrm{r}} T$. The module $\Gamma$ decomposes as $T \oplus(M, T)$. Since $\operatorname{Hom}_{\Gamma}(T, T)=\Lambda$ and $\Lambda$ is injective, it follows by Lemma 2.4 (b) that

$$
\operatorname{Hom}_{\Gamma}(T, C) \simeq \operatorname{Hom}_{\Lambda}((C, T),(T, T))=\operatorname{Hom}_{\Lambda}((C, T), \Lambda)
$$

for all modules $C$ in $\bmod \Gamma$. This implies that $\operatorname{Hom}_{\Gamma}(T, C)=0$ if and only if $\operatorname{Hom}_{\Gamma}(C, T)=0$. Since a simple module $S$ is in $\operatorname{soc} T$ if and only if $\operatorname{Hom}_{\Gamma}(S, T) \neq 0$ and $S$ is in $T / \underline{\mathrm{r} T}$ if and only if $\operatorname{Hom}_{\Gamma}(T, S) \neq 0$, it follows that $\operatorname{add} \operatorname{soc} T=\operatorname{add} T / \underline{\mathrm{r}} T$.

Next we prove the converse of Proposition 5.7.

Proposition 5.8 Let $\Gamma$ be a Gorenstein algebra with the following properties.

(1) $\Gamma=T \oplus Q$, where $T$ is the maximal injective summand of $\Gamma$.

(2) $\operatorname{id}_{\Gamma} \Gamma=2$.

(3) dom.dim $\Gamma=2$.

(4) $\operatorname{add} \operatorname{soc} T=\operatorname{add} T / \underline{\mathrm{r}} T$.

Then $\Lambda=\operatorname{End}_{\Gamma}(T)$ is a selfinjective artin algebra and ${ }_{\Lambda} T \simeq \Lambda \oplus M$, where $M \simeq D \operatorname{Tr} M$. 
Proof: Let $\Lambda=\operatorname{End}_{\Gamma}(T)$ and $F=F_{\text {add }_{\Lambda} T}$. By Proposition 5.5 the algebra $\Lambda$ is $F$-selfinjective and $\mathcal{P}(F)=\mathcal{I}(F)=\operatorname{add}_{\Lambda} T$.

Since $\mathcal{I}(\Lambda)$ is contained in add $T$, there exists a projective $\Gamma$-module $P$ such that $\operatorname{Hom}_{\Gamma}(P, T)=$ $D\left(\Lambda^{\mathrm{op}}\right)$. Using the same arguments as in the proof of Proposition 5.7 we have that add $P / \underline{\mathrm{r}} P=$ add $\operatorname{soc} T$. Since add $\operatorname{soc} T=\operatorname{add} T / \underline{\mathrm{r}} T$, it follows that add $P=\operatorname{add} T$. Therefore $\operatorname{Hom}_{\Gamma}(T, T)=\Lambda$ is in $\mathcal{I}(\Lambda)$ and $\Lambda$ is selfinjective. We have that $\mathcal{P}(F)=\operatorname{add} T=\mathcal{I}(F)$. We have that ${ }_{\Lambda} T=\Lambda \oplus M$, where $M$ has no nonzero projective summands. Then $\mathcal{I}(F)=\mathcal{I}(\Lambda) \cup$ add $D \operatorname{Tr} M$, so $M$ must be isomorphic to $D \operatorname{Tr} M$. This completes the proof of the proposition.

Remark: Let $\Lambda$ be a symmetric artin algebra and let $N$ be a $\Lambda$-module such that $N \simeq \Omega_{\Lambda}^{n}(N)$ for some $n$. Denote $N \oplus \Omega_{\Lambda}(N) \oplus \cdots \oplus \Omega_{\Lambda}^{n-1}(N)$ by $M$. Since $D \operatorname{Tr} \simeq \Omega_{\Lambda}^{2}$ for symmetric artin algebras, $M \simeq D \operatorname{Tr} M$. So $T=\Lambda \oplus M$ gives an example of the above construction. In particular, if $\Lambda=k[x] /\left(x^{t}\right)$ for a field $k$, then $\Omega_{\Lambda}^{2}$ is the identity. Therefore $\Gamma=\operatorname{End}_{\Lambda}(\Lambda \oplus M)$ for a $\Lambda$-module $M$ without any nonzero projective summands has all the properties in Proposition 5.7. In particular, group rings $\Lambda=k[G]$ for some finite group $G$ over a field $k$ are symmetric artin algebras. They are known to have modules $M$ with $M \simeq D \operatorname{Tr} M$ [1].

Let $\Gamma$ be a Gorenstein algebra with $\operatorname{dom} \cdot \operatorname{dim} \Gamma=\operatorname{id}_{\Gamma} \Gamma=2$. Then we have shown the $\Gamma=$ $\operatorname{End}_{\Lambda}(T)$ for some relative cotilting tilting module $T$ over an $F$-selfinjective algebra $\Lambda$. Moreover, $\operatorname{add}_{\Lambda} T=\mathcal{O}_{\Lambda} \cup$ add $M$, where $M \simeq D \operatorname{Tr} M$. Then three things can happen: (I) $\mathcal{O}_{\Lambda}=\mathcal{P}(\Lambda)=$ $\mathcal{I}(\Lambda)$, (II) the module $M$ is zero and (III) the module $M$ is nonzero and the subcategory $\mathcal{P}(\Lambda)$ is properly contained in $\mathcal{O}_{\Lambda}$. In the first case $\Lambda$ is selfinjective and we have characterized when $\Gamma$ is given this way in terms of properties of modules in $\bmod \Gamma$ above. We end this section by characterizing the second and third cases in terms of properties of modules in $\bmod \Gamma$. Thereby we classify all the artin Gorenstein algebras $\Gamma$ with $\operatorname{dom} \cdot \operatorname{dim} \Gamma=\operatorname{id}_{\Gamma} \Gamma=2$ into three different types. In order to give this structure theorem we need some preliminary results.

Proposition 5.9 Let $\Gamma$ be Gorenstein with $\operatorname{dom} \cdot \operatorname{dim} \Gamma=\operatorname{id}_{\Gamma} \Gamma=2$ and $\Gamma=T \oplus Q$, where $T$ is the maximal injective summand of $\Gamma$. Denote $\operatorname{End}_{\Gamma}(T)$ by $\Lambda$.

(a) The subcategory ${ }^{\perp} \Gamma$ has minimal left and right almost split maps and almost split sequences with translate $\tau_{\perp \Gamma}$.

(b) A morphism $f: B \rightarrow C$ is (minimal) right almost split in ${ }^{\perp} \Gamma$ if and only if $(f, T):(C, T) \rightarrow$ $(B, T)$ is (minimal) left almost split in $\bmod \Lambda$. A morphism $g: A \rightarrow B$ is (minimal) left almost split in ${ }^{\perp} \Gamma$ if and only if $(g, T):(B, T) \rightarrow(A, T)$ is (minimal) right almost split in $\bmod \Lambda$.

(c) An exact sequence $0 \rightarrow A \rightarrow B \rightarrow C \rightarrow 0$ in ${ }^{\perp} \Gamma$ is almost split in ${ }^{\perp} \Gamma$ if and only if $0 \rightarrow(C, T) \rightarrow(B, T) \rightarrow(A, T) \rightarrow 0$ is almost split in $\bmod \Lambda$ with $(C, T)$ not in $\operatorname{add}_{\Lambda} T$. In particular, $\operatorname{Tr} D(C, T) \simeq\left(\tau_{\perp_{\Gamma}} C, T\right)$ for $C$ indecomposable nonprojective in ${ }^{\perp} \Gamma$ and $\tau_{\perp_{\Gamma}}^{-1} \operatorname{Hom}_{\Lambda}(X, T) \simeq$ $\operatorname{Hom}_{\Lambda}(D \operatorname{Tr} X, T)$ for $X$ indecomposable not in $\mathcal{P}(F)$.

Proof: Let $\Gamma$ be Gorenstein with dom.dim $\Gamma=\operatorname{id}_{\Gamma} \Gamma=2$ and $\Gamma=T \oplus Q$, where $T$ is the maximal injective summand of $\Gamma$. Denote $\operatorname{End}_{\Gamma}(T)$ by $\Lambda$.

(a) Since $\Gamma$ is Gorenstein, the module $\Gamma$ is a cotilting module in mod $\Gamma$. Therefore by $[2$, Corollary 5.10] the subcategory ${ }^{\perp} \Gamma$ is functorially finite in $\bmod \Gamma$ and extension closed. By $[4$, Proposition ] the subcategory ${ }^{\perp} \Gamma$ has minimal right and left almost split homomorphisms and almost split sequences.

(b) We have that $\bmod \Lambda$ is dual to ${ }^{\perp} \Gamma$ via the functors $\operatorname{Hom}_{\Lambda}(, T): \bmod \Lambda \rightarrow \bmod \Gamma$ and $\operatorname{Hom}_{\Gamma}(, T): \bmod \Gamma \rightarrow \bmod \Lambda$ by $[6$, Corollary 3.6 (a)]. Let $f: B \rightarrow C$ be a right almost split map in ${ }^{\perp} \Gamma$. Then $(f, T):(C, T) \rightarrow(B, T)$. Let $X$ be a indecomposable module in $\bmod \Lambda$ and $g:(C, T) \rightarrow X$ a nonisomorphism. Then $X \simeq\left(X^{\prime}, T\right)$ for some module $X^{\prime}$ in ${ }^{\perp} \Gamma$ and $g=\left(g^{\prime}, T\right):(C, T) \rightarrow\left(X^{\prime}, T\right)$ for a map $g^{\prime}: X^{\prime} \rightarrow C$. The map $g^{\prime}$ is a nonisomorphism and $X^{\prime}$ is indecomposable. Since $f$ is right almost split, there exists a homomorphism $h: X^{\prime} \rightarrow B$ such that $g^{\prime}=f \circ h$. Hence, we have that $g=\left(g^{\prime}, T\right)=(h, T) \circ(f, T)$. Therefore $(f, T):(C, T) \rightarrow(B, T)$ is a left almost split homomorphism. Minimality is shown using the same type of arguments. To show the remaining parts of (b) similar arguments are used. 
(c) This follows directly from (b) and the fact that an almost split sequence $0 \rightarrow A \rightarrow B \rightarrow$

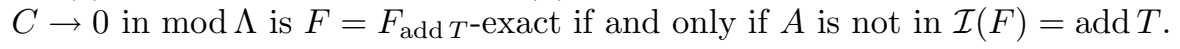

Let $\Gamma$ be Gorenstein with $\operatorname{dom} \cdot \operatorname{dim} \Gamma=\operatorname{id}_{\Gamma} \Gamma=2$ and $\Gamma=T \oplus Q$, where $T$ is the maximal injective summand of $\Gamma$. Denote $\operatorname{End}_{\Gamma}(T)$ by $\Lambda$. Then we know by Proposition 5.1 that $\operatorname{add}_{\Lambda} T=$ $\operatorname{add}\left\{(\operatorname{Tr} D)^{i} \Lambda\right\}_{i=0}^{\infty} \cup$ add $M$ for a module $M$ with $M \simeq D \operatorname{Tr} M$. The algebra $\Gamma$ is said to be minimal if $M=0$. Next we give a characterization of $\Gamma$ being minimal in terms of properties of modules in $\bmod \Gamma$.

Proposition 5.10 Let $\Gamma$ be Gorenstein with $\operatorname{dom} \cdot \operatorname{dim} \Gamma=\operatorname{id}_{\Gamma} \Gamma=2$ and $\Gamma=T \oplus Q$, where $T$ is the maximal injective summand of $\Gamma$. Denote $\operatorname{End}_{\Gamma}(T)$ by $\Lambda$.

(a) For all indecomposable noninjective projective $\Gamma$-modules $P$ there exists an exact sequence $0 \rightarrow P \stackrel{f}{\rightarrow} X \stackrel{g}{\rightarrow} P^{\prime}$, where $f$ is minimal left almost split in ${ }^{\perp} \Gamma, g$ is minimal right almost split ${ }^{\perp}{ }^{\perp} \Gamma$ and $P^{\prime}$ is an indecomposable projective $\Gamma$-module uniquely determined up to isomorphism. Denote $P^{\prime}$ by $\tau_{\perp_{\Gamma}}^{-1} P$. Moreover, if $P=\left(T_{0}, T\right)$, then $\tau_{\perp_{\Gamma}}^{-1} P \simeq\left(D \operatorname{Tr} T_{0}, T\right)$.

(b) The algebra $\Gamma$ is minimal if and only if $\tau_{\perp_{\Gamma}}^{-r} P$ is injective for all indecomposable noninjective projective $\Gamma$-modules $P$ for some $r$ in $\mathbf{N}$.

Proof: Let $\Gamma$ be Gorenstein with dom.dim $\Gamma=\operatorname{id}_{\Gamma} \Gamma=2$ and $\Gamma=T \oplus Q$, where $T$ is the maximal injective summand of $\Gamma$. Denote $\operatorname{End}_{\Gamma}(T)$ by $\Lambda$. The module ${ }_{\Lambda} T$ is a relative cotilting module with respect to the subfunctor $F=F_{\operatorname{add}_{\Lambda} T}$ of $\operatorname{Ext}_{\Lambda}^{1}($,$) , where \mathcal{P}(F)=\mathcal{I}(F)=\operatorname{add}_{\Lambda} T$. Moreover, $\Gamma \simeq \operatorname{End}_{\Lambda}(T)$.

(a) Let $P$ be an indecomposable noninjective projective $\Gamma$-module. Then $P \simeq \operatorname{Hom}_{\Lambda}\left(T_{0}, T\right)$ for some indecomposable nonprojective module $T_{0}$ in add ${ }_{\Lambda} T$. Let $0 \rightarrow D \operatorname{Tr} T_{0} \rightarrow E \rightarrow T_{0} \rightarrow 0$ be the almost split sequence ending in $T_{0}$ in $\bmod \Lambda$. Then the induced sequence $0 \rightarrow\left(T_{0}, T\right)=P \stackrel{f}{\rightarrow}$ $(E, T) \stackrel{g}{\rightarrow}\left(D \operatorname{Tr} T_{0}, T\right)$ is exact in $\bmod \Gamma$, where $f$ is minimal left almost split in ${ }^{\perp} \Gamma$ and $g$ is minimal right almost split in ${ }^{\perp} \Gamma$ by Proposition 5.9. Since $\Lambda$ is $F$-selfinjective, the indecomposable module $D \operatorname{Tr} T_{0}$ is again in add ${ }_{\Lambda} T$. Therefore the module $\left(D \operatorname{Tr} T_{0}, T\right)$ is an indecomposable projective $\Gamma$-module.

To complete the proof of (a) we need to prove that the module $P^{\prime}$ is uniquely determined up to isomorphism. Let $0 \rightarrow P=\left(T_{0}, T\right) \stackrel{f}{\rightarrow} X \stackrel{g}{\rightarrow} P^{\prime}$ be an exact sequence with the desired properties in (a). Then $\left(P^{\prime}, T\right) \stackrel{(g, T)}{\rightarrow}(X, T) \stackrel{(f, T)}{\rightarrow}(P, T)=T_{0} \rightarrow 0$ is exact in mod $\Lambda$ with $(g, T)$ minimal left almost split and $(f, T)$ minimal right almost split in $\bmod \Lambda$ by Proposition 5.9. Then it is easy to see that $(g, T)$ is a monomorphism, hence the induced sequence in $\bmod \Lambda$ is the almost split sequence ending in $T_{0}$. This shows that the module $P^{\prime} \simeq\left(D \operatorname{Tr} T_{0}, T\right)$ is uniquely determined up to isomorphism. The rest of (a) follows directly from this.

(b) Let $\Gamma, T, \Lambda$ and $F$ be as above. Assume that $\Gamma$ is minimal. Then $\operatorname{add}{ }_{\Lambda} T=\operatorname{add}\left\{(\operatorname{Tr} D)^{i} \Lambda\right\}_{i=0}^{\infty}$ by the definition of minimality. Let $P$ be an indecomposable noninjective projective $\Gamma$-module. Then $P \simeq\left(T_{0}, T\right)$ for some indecomposable nonprojective module $T_{0}$ in $\operatorname{add}_{\Lambda} T$. By (a) we have that $\tau_{\perp \Gamma}^{-r} P \simeq\left((D \operatorname{Tr})^{r} T_{0}, T\right)$. Since $(D \operatorname{Tr})^{i} X$ is projective for any module $X$ in add ${ }_{\Lambda} T$ for some $i$, it follows that $\tau_{\perp \Gamma}^{-r} P$ is injective for some $r$.

Conversely, if $\tau_{\perp_{\Gamma}}^{-r} P$ is injective for some $r$, it means that $(D \operatorname{Tr})^{r} T_{0}$ is projective. This implies that all indecomposable nonprojective modules $X$ in add ${ }_{\Lambda} T=\mathcal{P}(F)$ have the property that $(D \operatorname{Tr})^{i} X$ is projective for some $i$. Hence, we have that $\operatorname{add}_{\Lambda} T=\operatorname{add}\left\{(\operatorname{Tr} D)^{i} \Lambda\right\}_{i=0}^{\infty}$ and therefore $\Gamma$ is minimal.

Now we are ready to give the first part of the structure theorem for the Gorenstein algebras $\Gamma$ with dom.dim $\Gamma=\operatorname{id}_{\Gamma} \Gamma=2$.

Proposition 5.11 Let $\mathcal{E}$ denote the class of $D$ Tr-selfinjective artin algebras and $\mathcal{T}$ the class of artin Gorenstein algebras $\Gamma$ with $\operatorname{dom} \cdot \operatorname{dim} \Gamma=\operatorname{id}_{\Gamma} \Gamma=2$. Denote $\operatorname{add}\left\{(\operatorname{Tr} D)^{i} \Lambda\right\}_{i=0}^{\infty}$ by $\mathcal{O}_{\Lambda}$. Then there is an $1-1$ correspondence up to Morita equivalence between $\{(\Lambda, M) \mid \Lambda \in \mathcal{E}, M \simeq$ $D \operatorname{Tr} M, \mathcal{P}(\Lambda) \varsubsetneqq \mathcal{O}_{\Lambda} \cup$ add $\left.M\right\}$ and $\mathcal{T}$. The correspondence is given by $(\Lambda, M) \mapsto \operatorname{End}_{\Lambda}(C \oplus M)$ 
where $C$ is a $\Lambda$-module such that add $C=\mathcal{O}_{\Lambda}$ and $\Gamma \mapsto\left(\Lambda=\operatorname{End}_{\Gamma}(T), M\right)$ where the module $T$ is the maximal injective summand of $\Gamma$ and the module $M$ is such that add ${ }_{\Lambda} T$ is the disjoint union of $\mathcal{O}_{\Lambda}$ and add $M$.

Proof: We know from earlier results that the given correspondences are maps between the give classes. So it remains to prove that it is one-to-one up to Morita equivalence.

Let $\Lambda_{i}$ and $T_{i}$ be such that $\Lambda_{i}$ is $F_{i}=F_{\text {add } T_{i}}$-selfinjective with $\mathcal{P}\left(\Lambda_{i}\right)$ properly contained in add $T_{i}$ for $i=0,1$. Let $\Gamma$ be in $\mathcal{T}$ and assume that $\Gamma \simeq \operatorname{End}_{\Lambda_{i}}\left(T_{i}\right)$ for $i=0,1$. Let $I$ denote the maximal injective summand of $\Gamma$. Then we know by Proposition 4.1 that add $T_{\Gamma}=$ add $I$ for $i=0,1$. Moreover, we have that $\Lambda_{i} \simeq \operatorname{End}_{\Gamma}\left(T_{i}\right)$ for $i=0,1$. Hence if follows that $\Lambda_{0}$ and $\Lambda_{1}$ are Morita equivalent.

Conversely, let $\Lambda$ be a $D$ Tr-selfinjective algebra and $M$ a $\Lambda$-module such that $M \simeq D \operatorname{Tr} M$.

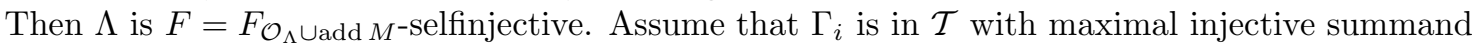
$T_{i}$ and that $\Lambda \simeq \operatorname{End}_{\Gamma_{i}}\left(T_{i}\right)$, where $M_{i}=M$ is such that add ${ }_{\Lambda} T_{i}$ is the disjoint union of $\mathcal{O}_{\Lambda}$ and add $M_{i}$ for $i=0,1$. Since $\mathcal{O}_{\Lambda} \cup$ add $M_{i}=\mathcal{P}(F)$ for $i=0,1$, we have that add ${ }_{\Lambda} T_{0}=\operatorname{add}_{\Lambda} T_{1}$. Moreover, $\Gamma_{i} \simeq \operatorname{End}_{\Lambda}\left(T_{i}\right)$ for $i=0,1$, so that $\Gamma_{0}$ and $\Gamma_{1}$ are Morita equivalent. This completes the proof of the proposition.

Finally we can give the division of the class $\mathcal{T}$ of artin Gorenstein algebras $\Gamma$ with dom.dim $\Gamma=$ $\operatorname{id}_{\Gamma} \Gamma=2$ into three different types.

Theorem 5.12 Let $\mathcal{T}$ be as above. Then an algebra $\Gamma$ in $\mathcal{T}$ is of one of the following three types.

(I) $\operatorname{add} T / \underline{\mathrm{r}} T=\operatorname{add} \operatorname{soc} T$, where $T$ is the maximal injective summand of $\Gamma$.

(II) $\Gamma$ is minimal.

(III) There is an indecomposable noninjective projective $\Gamma$-module $P$ such that $P$ is $\tau_{\perp} \Gamma^{-}$ periodic.

Moreover, if $\Gamma$ is of type (III), then we have that $\Gamma \simeq \operatorname{End}_{\Gamma^{\prime}}\left(\Gamma^{\prime} \oplus M\right)^{\mathrm{op}}$, where $\Gamma^{\prime}$ is of type (II) and $M$ is in ${ }^{\perp} \Gamma^{\prime}$ satisfying $M \simeq \tau_{\perp} \Gamma^{\prime}(M)$.

Proof: Let $\Gamma$ be an algebra in $\mathcal{T}$. If all indecomposable noninjective projective $\Gamma$-modules $P$ are $\tau_{\perp}$-periodic, then the corresponding pair $(\Lambda, M)$ has the properties that $\Lambda$ is selfinjective, $M \simeq D \operatorname{Tr} M$ and $\Gamma \simeq \operatorname{End}_{\Lambda}(\Lambda \oplus M)$. By Proposition 5.7 the algebra $\Gamma$ is of type (I).

Assume that not all indecomposable noninjective projective $\Gamma$-modules $P$ are $\tau_{\perp} \Gamma_{\text {-periodic. If }}$ there is no indecomposable noninjective projective $\Gamma$-module $P$ which is $\tau_{\perp} \Gamma_{\text {-periodic, then }} \Gamma$ is minimal and of type (II). Let $\Gamma=T \oplus Q_{0} \oplus Q_{1}$, where $T$ is the maximal injective summand of

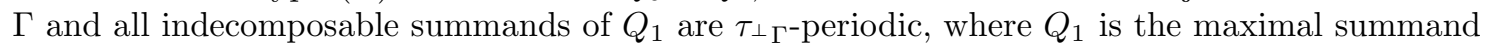
of $\Gamma$ with this property. Denote $\operatorname{End}_{\Gamma}(T)$ by $\Lambda$. Then $\operatorname{add}_{\Lambda} T=\mathcal{P}(\Lambda) \cup \operatorname{add}\left(Q_{0}, T\right) \cup \operatorname{add}\left(Q_{1}, T\right)$, where $\mathcal{O}_{\Lambda}=\mathcal{P}(\Lambda) \cup \operatorname{add}\left(Q_{0}, T\right)$. Let $M^{\prime}=\left(Q_{1}, T\right)$ and $T^{\prime}=\Lambda \oplus\left(Q_{0}, T\right)$. Then add $T^{\prime}=\mathcal{O}_{\Lambda}$. Denote $\operatorname{End}_{\Lambda}\left(T^{\prime}\right)$ by $\Gamma^{\prime}$. Then $\Gamma^{\prime}$ is minimal and the module $M=\left(M^{\prime}, T^{\prime}\right)$ has the property that $M \simeq \tau_{\perp} \Gamma^{\prime}(M)$ by Proposition 5.9. Then we have that

$$
\operatorname{End}_{\Gamma^{\prime}}\left(\Gamma^{\prime} \oplus\left(M^{\prime}, T^{\prime}\right)\right)^{\mathrm{op}} \simeq \operatorname{End}_{\Gamma^{\prime}}\left(\left(M^{\prime} \oplus T^{\prime}, T^{\prime}\right)\right)^{\mathrm{op}} \simeq \operatorname{End}_{\Lambda}\left(M^{\prime} \oplus T^{\prime}\right) \simeq \operatorname{End}_{\Lambda}\left({ }_{\Lambda} T\right) \simeq \Gamma
$$

and we are done.

\section{References}

[1] J. L. Alperin, Periodicity in groups, Illinois J. Math. 21 (1977) no. 4, 776-783.

[2] M. Auslander, I. Reiten, Applications of homologically finite subcategories, Adv. in Math., 86 (1991) 111-152.

[3] M. Auslander, I. Reiten, Syzygy modules over artin algebras, in preparation. 
[4] M. Auslander, S. O. Smalø, Almost split sequences in subcategories, J. Algebra 69 (1981) 426-454; Addendum J. Algebra 71 (1981) 592-594.

[5] M. Auslander, Ø. Solberg, Relative homology and representation theory I, Relative homology and homologically finite subcategories.

[6] M. Auslander, Ø. Solberg, Relative homology and representation theory II, Relative cotilting theory.

[7] M. Auslander, Ø. Solberg, Relative homology and representation theory III, Cotilting modules and Wedderburn correspondence. 